\title{
Distributed multi-munition cooperative guidance based on clock synchronization for switching and noisy networks
}

\author{
Sheng $\mathrm{Yi}^{1,2} \cdot$ Xiaoyu She ${ }^{1}$. Dong Guo ${ }^{3} \cdot$ Tong Zhao $^{4} \cdot$ Chengwei Yang ${ }^{1} \cdot \mathrm{Jie} \mathrm{Li}^{1}$ \\ Published online: 31 March 2020 \\ (C) The Author(s) 2020
}

\begin{abstract}
This paper proposes a novel three-dimensional guidance method based on clock synchronization algorithms, creatively solving the problems of randomized change, non-connection and even communication outage caused by packet loss and delay in the co-guidance of loitering munitions. The proposed method is characterized by fast convergence of the aspect angle rate in the longitudinal plane, and the attack time constrains in the lateral plane. Normal accelerations on the two sub-planes are thereby calculated, so as to control the munitions' co-attack. Simulation experiments show that the proposed method can ensure reliable and stable asymptotic agreement of attack time expectation for the cooperative multiple munitions under the switching and noisy networks.
\end{abstract}

Keywords Loitering munition · Cooperative guidance $\cdot$ Clock synchronization · Distributed algorithm

\section{Introduction}

Loitering munition is a kind of new concept munition which can perform "cruising flight" and "standby" above the target area and perform a variety of operational missions. It is the product of organic combination of UAV technology and munition technology and can realize reconnaissance and damage assessment, precision strike, communication, relay, target indication, airborne alert and other combat tasks. The

Chengwei Yang

yangchengwei@bit.edu.cn

1 School of Mechatronical Engineering, Beijing Institute of Technology, Beijing 100081, China

2 Beijing Special Electromechanical Research Institute, Beijing 100081, China

3 School of Automation Science and Electrical Engineering, Beihang University, Beijing 100191, China

4 School of Mathematical Sciences, University of Chinese Academy of Sciences, Beijing 100190, China 
coordinated attack of multiple loitering munitions can greatly improve the efficiency of striking target $[1,2]$. Multiple loitering munitions can cooperate and coordinate with each other through information sharing and jointly complete the strike task, which greatly enhances the strike ability of loitering munition and increases the probability of penetration and destroying target by loitering munition [3].

Simultaneous arrival is a key issue in the study of coordinated attack of multiple loitering munitions, which can be attributed to the consistency of guidance time [4]. The consistency problem of guidance time mainly studies the information interaction mode between each munition in the coordinated attack system, so that the guidance time of all munitions tends to the same value, so as to achieve the maximum damage efficiency to the target. Some research achievements have been presented for this purpose. Jeon et al. proposed a homing guidance law called cooperative proportional guidance $(\mathrm{CPN})$ for several for one participation scenario of typical anti-ship missile. This method has the flexibility to allow different speeds to cooperate and attack simultaneously [5]. Kumar et al. proposed an impact time guidance law based on sliding mode control. Even for large course angle error and negative initial closure speed, the desired impact time is achieved by applying sliding mode on the switching surface based on collision route and estimated time thought [6]. In the literature [7], a new composite guidance law composed of two phases for impact time control is proposed. The first phase is to guide the missile to a suitable switching state for the second phase. And the second phase, which is derived from Lyapunov stability condition, can ensure the convergence of the relative range and heading error. In the literature [8], in order to consider the multiple constraints and time-variant velocity basically effectively, two closed-loop cooperative guidance methods are developed, by employing the efficient convex optimization technique and receding horizon control (RHC) strategy.

The above cooperative guidance methods are divided into centralized mode and distributed mode by the communication topology. Because the centralized communication topology also has the defects of poor robustness, high communication cost and bad extension [9], it is difficult to guarantee the centralized communication between munitions in the actual battlefield. Therefore, the distributed communication topology is often adopted, that is, through the local communication between adjacent munitions, the cooperative guidance for the cognitive consistency of the cooperative target is achieved gradually.

In recent years, some researches on distributed cooperative guidance methods have achieved good results. Based on the centralized cooperative guidance law, Zhao and Zhou [10] selected the expected coordination variable as the average value of all missile residual time in the communication range to achieve the cooperative guidance. For the problem of cooperative guidance that needs to limit collision angle and attack static target simultaneously in the plane, Wang et al. [11] designed the cooperative bias proportional guidance law that can realize collision angle constraint based on the bias proportional guidance law with collision angle constraint [12] and taking the remaining time as the coordination variable. Song et al. [13] removed the bias term coefficient on the basis of the literature [3] for the static target in the plane and designed an expression of expected remaining time for time dispersion. By determining the appropriate expression coefficient, the system covariance stability is achieved under the condition of topological structure circulation. 
In order to meet the overload limit, Hou et al. [14] took the remaining time variance of zero as the cooperative target for the static target in the plane and designed a proportional guidance law of variable coefficient that could realize the cooperation in the limited time. For static targets in 3D space, Wei et al. [15] found the relationship between the missile remaining time and the proportional guidance coefficient based on the expert system containing two limit learning machines when the communication topology is strongly connected between missiles. Therefore, by adjusting the proportional guidance coefficient, the remaining time of missile is close to the maximum value of its communication missile, and coordinated attack is realized. However, Daughtery and Qu [16] designed the cooperative guidance law to optimize the required control energy based on the optimal cooperative control method and the cooperative remaining time estimation method, taking the distance and speed of the missile relative to the target as the coordination variables.

The above design of the multi-munition cooperative guidance law assumes that the communication between each munition is under ideal conditions, that is, the topology of the actual communication link is fixed. However, during the long flight, it is very likely that the communication packet loss will occur due to the interference of the complex battlefield environment, and the random delay caused by information transmission cannot be avoided. Therefore, the effect of time-varying topology on multi-munition cooperation must be considered in the design of multi-munition cooperative guidance law. In the literature [17], the problem of multi-missile cooperative guidance under the condition of topology uncertainty and communication delay is considered. The asymptotic stability of the guidance time divergence system ensures the consistent encounter time of multi-missile cooperative guidance. In the literature [18], the multi-missile cooperative guidance problem of the leading topology with hopping and delay in the communication topology is considered. It transforms the leading consistency problem into the stability problem of the error system. The guidance law is obtained by feedback linearization. The literature $[19,20]$ developed a two-step guidance strategy to deal with multi-missile distributed group cooperative guidance (DGCG) with fixed and switched directional communication topology to achieve group cooperative attack.

In the above research, although the communication topology is time varying, it is required to be connected at any time between munitions. Because of communication packet loss and time delay, the actual communication topology between munitions changes randomly and there are communication information noises. At some point, the communication topology is not connected or even the communication is interrupted between munitions. Therefore, how to solve the problem of multi-munition cooperative guidance of interrupted communication due to communication packet loss becomes the research content of this paper.

In this paper, multi-munition cooperative guidance for fixed target is studied. The time synchronization algorithm is used to solve the problem that the munition communication topology changes randomly, topology is disconnected and communication is interrupted due to packet loss and delay in the cooperative guidance process. Based on this, a time-constraint three-dimensional guidance method is designed. The specific strategy is to design the guidance law in longitudinal and lateral planes, respectively. In the longitudinal plane, it designs the guidance law of fast convergence of aspect 


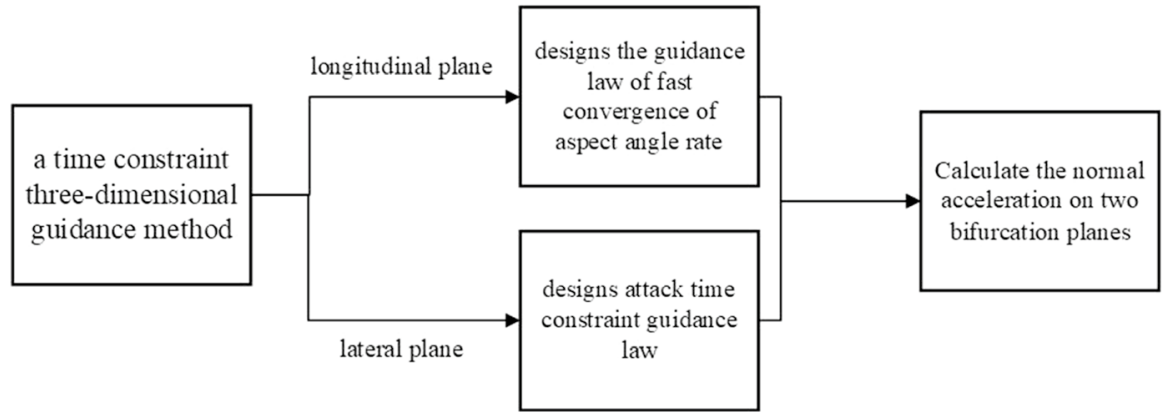

Fig. 1 Overall flow diagram

angle rate, and in the lateral plane, it designs attack time-constraint guidance law. After the guidance law is designed in two directions, the normal acceleration on two bifurcation planes can be calculated, and then, the loitering munition can be controlled to attack the target cooperatively. The overall flow diagram is shown in Fig. 1.

Other parts of this paper are organized as follows. In the second chapter, basic assumptions involved in this algorithm are briefly introduced, and the model of the researched problem is established. The main content of the algorithm is introduced in the third chapter. The fourth chapter is the corresponding simulation test. Finally, the conclusion is presented in the fifth chapter.

\section{Modeling of problems}

This paper mainly studies the problem of time consistency cooperative guidance of loitering munition for ground fixed targets in three-dimensional space. To facilitate analysis, the following hypothesis is made:

1. Ignoring the impact of earth rotation and curvature on ammunition;

2. Ammunition is rigid and flies at a constant rate, ignoring aerodynamic disturbance.

According to the above hypothesis, the kinematics equation of loitering munition in three-dimensional space is obtained:

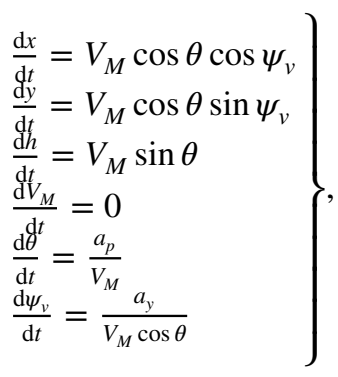


where $(x, y, h)$ is the position coordinates of loitering munition in the inertial coordinate system; $V_{M}$ is the velocity; $\theta$ and $\psi_{v}$ are trajectory inclination angle and deflection angle, respectively; $m$ is the munition mass; and $a_{p}$ and $a_{y}$ are, respectively, the longitudinal and lateral overload of munition, as shown in Fig. 2.

\section{3D guidance method design}

A time-constraint three-dimensional guidance method is designed in this paper. The specific strategy is to design the guidance law in longitudinal and lateral planes, respectively. In the longitudinal plane, it designs the guidance law of fast convergence of aspect angle rate, and in the lateral plane, it designs attack time-constraint guidance law. After the guidance law is designed in two directions, the normal acceleration on two bifurcation planes can be calculated.

\subsection{Design of longitudinal plane guidance law}

We hope that the aspect angle rate on the longitudinal plane can rapidly converge and remain close to 0 , which can not only guarantee that the munition has a relatively straight trajectory and a small miss distance on the longitudinal plane, but also has little interference on the lateral plane, so as to guarantee a good attack time control effect. Therefore, the following sliding surface is selected:

$$
S_{\text {lon }}=P \cdot \dot{q}_{\theta},
$$

where $P$ is the normal number and $\dot{q}_{\theta}$ is the aspect angle rate of the longitudinal plane between munition and target. Select the power reaching law with better convergence effect:

$$
\dot{S}_{\text {lon }}=-Q \cdot\left|S_{\text {lon }}\right|^{R} \operatorname{sgn}\left(S_{\text {lon }}\right),
$$

Fig. 2 Three-dimensional relationship of relative motion between munition and target. Here, $M$ is the munition position; $r$ is the relative distance between munition and target $p$; $T$ is the fixed target position; $q_{\theta}$ is the longitudinal aspect angle of munition; $q_{\psi}$ is the lateral aspect angle of munition; $\psi_{v}$ is trajectory deflection angle; $\theta$ is the trajectory inclination angle

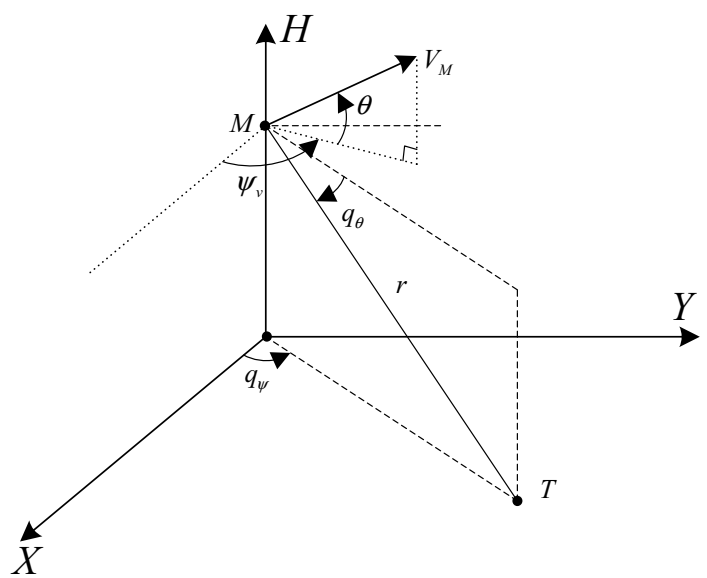


where $Q$ is the normal number, $R$ is the index in the power reaching law and the size is $0<R<1$. The guidance law of the munition on the longitudinal plane can be obtained from Eqs. (2) and (3):

$$
a_{p}=\frac{r\left(-\frac{2 \dot{r} \dot{q}_{\theta} P}{r}+Q\left|S_{\mathrm{lon}}\right|^{R} \operatorname{sgn}\left(S_{\mathrm{lon}}\right)\right)}{P \cos \eta},
$$

where $\eta$ is the advance angle, that is, the difference between the munition course angle and the aspect angle on the corresponding plane.

\subsection{Design of lateral plane guidance law}

The lateral plane not only hopes the miss distance of the munition to be zero, but also requires the actual attack time to be equal to the expected attack time. The lateral guidance law is designed by using the sliding mode variable structure control method with strong robustness. The design of sliding surface must take both miss distance and attack time into consideration. Therefore, the sliding surface adopts the combination of aspect angle rate and attack time error:

$$
\begin{aligned}
S_{\text {lat }} & =\frac{\dot{q}_{\psi}}{c_{q}}+\frac{e}{c_{t}} \operatorname{sgn}\left(\dot{q}_{\psi}\right) \\
& =\frac{\dot{q}_{\psi}}{c_{q}}+\frac{\left(t+t_{\mathrm{go}}-t *\right)}{c_{t}} \operatorname{sgn}\left(\dot{q}_{\psi}\right),
\end{aligned}
$$

where $c_{q}$ and $c_{t}$ are the dimensionless parameters; $\dot{q}_{\psi}$ is the aspect angle rate in the lateral plane between munition and target; $e=t+t_{\mathrm{go}}-t^{*}$ is the attack time error; $t^{*}$ is the expected attack time; and $t_{\text {go }}$ is the estimation of the remaining flight time of munition. Its expression is:

$$
t_{\mathrm{go}}=\frac{r}{V_{\mathrm{M}}}\left(1+\frac{\eta^{2}}{2(2 N-1)}\right),
$$

where $N>1$ is the effective navigation ratio and $\eta$ is the advance angle, that is, the difference between the munition course angle and the aspect angle on the corresponding plane.

As can be seen from Eq. (5), the first term of the sliding mode surface plays the role of convergence of the aspect angle rate and the second term plays the role of controlling the actual attack time approaching to the expected attack time. If the system can enter the sliding mode and be stable in the sliding mode area within the limited time, the munition can strike the target with a small miss distance and attack time error. The controller is designed by equivalent sliding mode algorithm, which is divided into two parts: the equivalent controller and the discontinuous controller:

$$
a_{y}=a_{y}^{c}+a_{y}^{d} .
$$


When the system enters sliding mode, the equivalent controller $a_{y}^{c}$ maintains the control system in sliding mode. When $\dot{S}_{\text {lat }}=0$, the expression of the equivalent controller is obtained:

$$
\begin{aligned}
a_{y}^{c}= & \left\{-\frac{2 \dot{r} \dot{q}_{\psi}}{c_{q} r}+\frac{1}{c_{t}} \operatorname{sgn}\left(\dot{q}_{\psi}\right)\right. \\
& \left.\times\left[1+\frac{\dot{r}}{V_{\mathrm{M}}}\left(1+\frac{\eta^{2}}{2(2 N-1)}\right)-\frac{r \eta \dot{q}_{\psi}}{V_{\mathrm{M}}(2 N-1)}\right]\right\} \\
& \times\left(\frac{1}{c_{q}} \frac{\cos \eta}{r}-\frac{1}{c_{t}} \frac{r \eta \operatorname{sgn}\left(\dot{q}_{\psi}\right)}{V_{\mathrm{M}}^{2}(2 N-1)}\right)^{-1} .
\end{aligned}
$$

When the system does not enter the sliding mode, the design of discontinuous controller $a_{y}^{d}$ enables the system to rapidly approach the sliding mode, and the expression of the controller is

$$
a_{y}^{d}=k \cdot \operatorname{sgn}\left(S_{\text {lat }}\right)
$$

where the value of gain $k$ is:

$$
k=W \cdot\left[\operatorname{sgn}\left(\frac{\cos \eta}{c_{q} r}-\frac{r \eta \operatorname{sgn}\left(\dot{q}_{\psi}\right)}{c_{t} V_{M}^{2}(2 N-1)}\right)\right]^{-1},
$$

where $W$ is normal number.

In order to reduce the chattering phenomenon of the guidance law, the symbol function of the discontinuous controller can be changed into sgmf function:

$$
\operatorname{sgmf}\left(S_{\text {lat }}\right)=2\left(\frac{1}{1+e^{-b S_{\text {lat }}}}-\frac{1}{2}\right),
$$

where $b>0$.

\section{Distributed clock synchronization}

\subsection{Distributed clock synchronization algorithm for switching and noisy networks}

We write the local time of each agent $i$ as $\tau_{i}(t)=t+\alpha_{i}$, where $\alpha_{i}$ is a constant denoting the time drift of agent $i$. Here, we assume the clock frequency of each agent is accurate in a short time. We aim to design a distributed protocol such that all the agents have a same drift. We design our algorithm as follows. Let $\hat{\alpha}_{j}(l)$ be the drift correction factor of agent $j$ which is initially set to be zero. Let $T$ be a constant denoting the period and $\beta_{j}$ be the real time of the protocol start for agent $j$. Without loss of generality, we assume 


$$
0 \leq \beta_{1}<\beta_{2}<\cdots<\beta_{n}<T .
$$

Each agent $j$ periodically broadcasts its local time plus correction $l T+\beta_{j}+\alpha_{j}+\hat{\alpha}_{j}(l), l=0,1, \ldots$, to all other agents. Set $T_{j}^{l}:=l T+\beta_{j}$ to be the real sending time of this information. The neighbors of agent $j$ have a probability to successfully receive this information and record their local receiving time. Let $R_{i}^{j, l}$ and $\tau_{i}^{j, l}$ denote the received information and receiving local time of agent $i$ during its local time interval $\left[T_{i}^{l}+\alpha_{i}, T_{i}^{l+1}+\alpha_{i}\right)$, respectively, if the information comes from agent $j$. Because the sending time of agent $i$ and the receiving time of agent $j$ may belong to different local periods, we have

$$
\tau_{i}^{j, l}=S_{i}^{j, l}+d_{i}^{j, l}+\alpha_{i} \in\left[T_{i}^{l}+\alpha_{i}, T_{i}^{l+1}+\alpha_{i}\right),
$$

where $S_{i}^{j, l}$ denotes the real sending time and $d_{i}^{j, l}$ denote the communication delay from $j$ to $i$. The time delay in wireless networks includes sender processing delay, media access delay, transmit time, radio propagation time and receive process time. Because the transmission distance of wireless signal by sensor nodes or robots is not long whose radio propagation time is negligible, and if an information has been not correctly received we will discard it without retransmission, the delay is very short and almost a fixed value but with small fluctuation whose expectation can be measured beforehand. Then, assume $\overline{d_{i}^{j}}:=E d_{i}^{j, l}$ is known. Meanwhile, the received information

$$
R_{i}^{j, l}=S_{i}^{j, l}+\alpha_{j}+\hat{\alpha}_{j}\left(\left(S_{i}^{j, l}-\beta_{j}\right) / T\right)+\xi_{i}^{j, l},
$$

where $\xi_{i}^{j, l}$ denotes the communication noise.

Let $\mathcal{N}_{i}^{d}=\{j$ : Agent $i$ successfully receives the time information of agent $j$ in the interval $\left.\left[T_{i}^{l}, T_{i}^{l+1}\right)\right\}$, i.e.,

$$
\mathcal{N}_{i}^{l}=\left\{j: \tau_{i}^{j, l} \in\left[T_{i}^{l}+\alpha_{i}, T_{i}^{l+1}+\alpha_{i}\right)\right\} .
$$

Each agent $i$ updates its drift correction factor at the end of every period $\left[T_{i}^{l}, T_{i}^{l+1}\right)$ by

$$
\begin{aligned}
\hat{\alpha}_{i}(l+1) & =\hat{\alpha}_{i}(l)+a(l) \sum_{j \in N_{i}^{l}}\left(R_{i}^{j, l}-\tau_{i}^{j, l}-\hat{\alpha}_{i}(l)+\overline{d_{i}^{j}}\right) \\
& =\hat{\alpha}_{i}(l)+a(l) \sum_{j \in N_{i}^{l}}\left(\alpha_{j}+\hat{\alpha}_{j}\left(\left(S_{i}^{j, l}-\beta_{j}\right) / T\right)+\xi_{i}^{j, l}-d_{i}^{j, l}-\alpha_{i}-\hat{\alpha}_{i}(l)+\overline{d_{i}^{j}}\right),
\end{aligned}
$$

where the second equality uses (13) and (14) and $a(\cdot)$ is a predetermined gain function.

In summary, our algorithm for each agent $i$ can be described as follows: 
Algorithm 1 A distributed algorithm of clock synchronization underswitching and noisy networks

Input: $T$ : period; $L:$ maximum iteration number; $\alpha_{i}:$ time $\operatorname{drift} ; \hat{\alpha}_{i}(l) \operatorname{drift}$ correction factor; $\beta_{i}$ : real start time of the algorithm of agent $i ; a(l)$ : gain function; $\overline{d_{i}^{j}}$ : expectation of the communication delay from agents $j$ to $i$;

1: Initial: $\hat{\alpha}_{i}(0)=0$;

2: for $l=0 ; l<L ; l++$ do

3: Agent $i$ broadcasts the quantified information of $T_{i}^{l}+\alpha_{i}+\hat{\alpha}_{i}(l)$ to its neighbors at its local time $T_{i}^{l}+\alpha_{i}$, where $T_{i}^{l}:=l T+\beta_{i}$ denotes the real time;

4: Agent $i$ receives the information from its neighbors during its local time interval $\left[T_{i}^{l}+\alpha_{i}, T_{i}^{l+1}+\alpha_{i}\right)$, and records the received information and receiving local time as $R_{i}^{j, l}$ and $\tau_{i}^{j, l}$ respectively, if the information comes from agent $j$

5: At the end of the time interval $\left[T_{i}^{l}+\alpha_{i}, T_{i}^{l+1}+\alpha_{i}\right)$, update the drift correction factor by $\hat{\alpha}_{i}(l+1)=\hat{\alpha}_{i}(l)+a(l) \sum_{j \in N_{i}^{l}}\left(R_{i}^{j, l}-\tau_{i}^{j, l}-\hat{\alpha}_{i}(l)+\overline{d_{i}^{j}}\right)$, where $\quad N_{i}^{l}$ is defined by (15).

6: end for

Output: $t+\alpha_{i}+\hat{\alpha}_{i}(L)$ (local time + time drift correction factor)

\section{Remark 1}

1. Algorithm 1 does not need any global information.

2. Throughout Algorithm 1, each node only knows its local time and does not know the real time and the values of the parameters $\alpha_{i}$ and $\beta_{i}$. 


\subsection{Convergence of the algorithm}

We will show Algorithm 1 converges to clock synchronization, which means $\alpha_{i}+\hat{\alpha}_{i}(L)-\alpha_{j}-\hat{\alpha}_{j}(L) \rightarrow 0$ as $L \rightarrow \infty$ in probability sense.

(A1) There are constants $\gamma \in(1 / 2,1]$ and $0<c_{1} \leq c_{2}<\infty$ such that the deterministic sequence $\{a(l)\}_{l \geq 0}$ satisfies $a(0)>0$, and $c_{1} l^{-\gamma} \leq a(l) \leq c_{2} l^{-\gamma}$ for $l \geq 1$.

We define the $\sigma$-algebra generated by the noises and the delays $\xi_{i}^{j, l}, d_{i}^{j, l}, 0 \leq l \leq s, 1 \leq i \leq n, j \in \mathcal{N}_{i}^{l}$ and the delays by

$$
\mathcal{F}_{s}=\sigma\left(\xi_{i}^{j, l}, d_{i}^{j, l}, 0 \leq l \leq s, 1 \leq i \leq n, j \in \mathcal{N}_{i}^{l}\right) .
$$

The probability space of Algorithm 1 is written to be $\left(\Omega, \mathcal{F}_{\infty}, P\right)$.

(A2) $\left\{\sum_{j \in N_{i}^{l}}\left(\xi_{i}^{j, l}-d_{i}^{j, l}+d_{i}^{j}\right)\right\}_{1 \leq i \leq n, l \geq 0}$ are independent zero-mean random variables with uniformly bounded variance. Also, for any $l \geq 0,1 \leq i \leq n$ and $j \in \mathcal{N}_{i}$, the delay $d_{i}^{j, l}$ satisfies

$$
d_{i}^{j, l}<\left\{\begin{array}{lll}
T+\beta_{i}-\beta_{j}, & \text { if } \quad j>i \\
\beta_{i}-\beta_{j}, & \text { if } \quad j<i
\end{array} .\right.
$$

We also need introduce some definitions on graph theory. Let $G=\{V, E\}$ be a digraph, where $V=\{1,2, \ldots n\}$ is the set of nodes with node $i$ representing the $i$ th node and $E$ is the set of edges. An edge in $G$ is denoted by an ordered pair $(j, i)$, and $(j, i) \in E$ if and only if the $i$ th node can receive information from the $j$ th node directly. A sequence $\left(i_{1}, i_{2}\right),\left(i_{2}, i_{3}\right), \ldots,\left(i_{k-1}, i_{k}\right)$ of edges is called a directed path from node $i_{1}$ to node $i_{k}$. The $G$ is called a strongly connected digraph, if for any $i, j \in V$, there is a directed path from $i$ to $j$. For graphs $G(t)=\{V, E(t)\}, i \leq t<j$, their union is defined by $\cup_{i \leq t<j} \mathcal{G}(t):=\left\{\mathcal{V}, \cup_{i \leq t<j} \mathcal{E}(t)\right\}$. Note that there may exist multi-edges from one vertex to another in $\cup_{i \leq t<j} \mathcal{G}(t)$.

(A3) There exist a constant $l^{*} \geq 1$ and an infinite sequence $0=l_{0}<l_{1}<l_{2}<\ldots$ such that $l_{k}-l_{k-1} \leq l^{*}$ and $\left\{\mathcal{V}, \quad \cup_{l_{k-1} \leq l<l_{k}}\left\{(j, i): i \in \mathcal{V}, j \in \mathcal{N}_{i}^{l}\right\}\right\}$ is strongly connected for all $k \geq 1$.

Theorem 3.1 Consider Algorithm 1 satisfying (A1), (A2) and (A3). Then, the clock synchronization is asymptotically reached in mean square, i.e.,

$$
\lim _{L \rightarrow \infty} E\left|\alpha_{i}+\hat{\alpha}_{i}(L)-\alpha^{*}\right|^{2}=0,
$$

for some $\alpha^{*}$ and for all $1 \leq i \leq n$.

Proof of Theorem 3.1 To analyze Algorithm 1, we need transform it to a consensus protocol. With the assumption (12), we order the time sequence $T+\beta_{1}, \ldots, T+\beta_{n}, 2 T+\beta_{1} \ldots, 2 T+\beta_{n}, \ldots l T+\beta_{1}, \ldots, l T+\beta_{n} \ldots$ and record them as $t_{1}, t_{2}, t_{3}, \ldots$. Define $t_{0}:=0$. Then, for $l \geq 1$ we have $T_{i}^{l}=l T+\beta_{i}=t_{(l-1) n+i}$ and $t_{k}=T_{k-\left\lfloor\frac{k-1}{n}\right\rfloor n}^{\left\lfloor\frac{k-1}{n}\right\rfloor+1}$.

For $1 \leq i \leq n$ and $t \geq 0$, define 


$$
\tilde{\alpha}_{i}(t):=\left\{\begin{array}{lll}
0, & \text { if } & t \in\left[0, T+\beta_{i}\right) \\
\hat{\alpha}_{i}(l), & \text { if } & t \in\left[l T+\beta_{i},(l+1) T+\beta_{i}\right)
\end{array} .\right.
$$

Then, $\left\{\tilde{\alpha}_{i}(t)\right\}$ update their values at time $t_{1}, t_{2}, \ldots$ by Algorithm 1 . With (18), at time $t_{k}$ only node $k-\left\lfloor\frac{k-1}{n}\right\rfloor n$ updates the value of $\left\{\tilde{\alpha}_{i}(t)\right\}$, but the other nodes keep $\left\{\tilde{\alpha}_{i}(t)\right\}$ unchanged. Thus, for $1 \leq i \leq n$ and $k \geq 1$, by (16) we have

$$
\begin{aligned}
\tilde{\alpha}_{i}\left(t_{k}\right)= & \tilde{\alpha}_{i}\left(t_{k-1}\right) \\
& +a(\lfloor(k-1) / n\rfloor) \sum_{j \in \tilde{N}_{i}(k-1)}\left(\alpha_{j}+\tilde{\alpha}_{j}\left(S_{i}^{j,\lfloor(k-1) / n\rfloor}\right)\right. \\
& \left.+\xi_{i}^{j,\lfloor(k-1) / n\rfloor}-d_{i}^{j,\lfloor(k-1) / n\rfloor}-\alpha_{i}-\tilde{\alpha}_{i}\left(t_{k-1}\right)+\overline{d_{i}^{j}}\right),
\end{aligned}
$$

where

$$
\tilde{\mathcal{N}}_{i}(k-1):= \begin{cases}\mathcal{N}_{i}^{\left\lfloor\frac{k-1}{n}\right\rfloor}, & \text { if } i=k-\left\lfloor\frac{k-1}{n}\right\rfloor n \\ \phi, & \text { otherwise }\end{cases}
$$

According to (18) and Algorithm 1, $S_{k-\left[\frac{k-1}{n}\right\rfloor n}^{j,\lfloor(k-1) / n\rfloor}$ is the sending time of the node $j$ 's information received by the node $k-\left\lfloor\frac{k-1}{n}\right\rfloor n$ during the time $\left[t_{k-n}, t_{k}\right)$. Because we assume time delays are small, which satisfies (17), we can get

$$
t_{k-n}<\underset{k-\left\lfloor\frac{k-1}{n}\right\rfloor n}{S^{\lfloor,(k-1) / n\rfloor}} \leq t_{k-1},
$$

which indicates

$$
\tilde{\alpha}_{j}\left(\begin{array}{c}
\left.S_{k-\left\lfloor\frac{k-1}{n}\right\rfloor n}^{\lfloor(k-1) / n\rfloor}\right) \\
\text { n }
\end{array} \tilde{\alpha}_{j}\left(t_{k-1}\right),\right.
$$

because the next updating time of node $j$ is $S_{k-\left\lfloor\frac{k-1}{n}\right\rfloor n}^{j\lfloor(k-1) / n\rfloor}+T>t_{k}$.

Set $x_{i}(k):=\alpha_{i}+\tilde{\alpha}_{i}\left(t_{k}\right), b(k):=a(\lfloor k / n\rfloor)$, and $\omega_{i}^{j, k}:=\xi_{i}^{j,\lfloor k / n\rfloor}-d_{i}^{j,\lfloor k / n\rfloor}+\overline{d_{i j}^{j}}$.

By (19), (20) and (21), we can get

$$
\begin{array}{r}
x_{i}(k)=x_{i}(k-1)+b(k-1) \\
\sum_{j \in \tilde{N}_{i}(k-1)}\left(x_{j}(k-1)+\omega_{i}^{j, k-1}-x_{i}(k-1)\right) .
\end{array}
$$

Huang investigates the convergence of the system (22) under the following assumptions [21]:

(A1') There are constants $\gamma^{\prime} \in(1 / 2,1]$ and $0<c_{1}^{\prime} \leq c_{2}^{\prime}<\infty$ such that the deterministic sequence $\{b(k)\}_{k \geq 0}$ satisfies $b(0)>0$, and $c_{1}^{\prime} k^{-\gamma^{\prime}} \leq b(k) \leq c_{2}^{\prime} k^{-\gamma^{\prime}}$ for $k \geq 1$. 
(A2') $\left\{\left(\sum_{j \in \tilde{\mathcal{N}}_{1}(k-1)} \omega_{1}^{j, k-1}, \ldots, \sum_{j \in \tilde{\mathcal{N}}_{n}(k-1)} \omega_{n}^{j, k-1}\right)\right\}_{k \geq 1}$ is as sequence of independent zeromean random vectors with uniformly bounded variance.

(A3') There exist a constant $k^{*} \geq 1$ and an infinite sequence $0=k_{0}<k_{1}<k_{2}<\ldots$ such that $k_{s}-k_{s-1} \leq k^{*}$ and $\left\{\mathcal{V}, \cup_{k_{s-1} \leq k<k_{s}}\left\{(j, i): i \in \mathcal{V}, j \in \tilde{\mathcal{N}}_{i}(k)\right\}\right\}$ is strongly connected for all $s \geq 1$.

Lemma 3.1 (Theorem 14 in [21]) Consider system (22) satisfying (A1'), (A2') and $\left(A 3^{\prime}\right)$. Then, the consensus is asymptotically reached in mean square, i.e.,

$$
\lim _{k \rightarrow \infty} E\left|x_{i}(k)-x^{*}\right|^{2}=0
$$

for some $x^{*}$ and for all $1 \leq i \leq n$.

By Lemma 3.1, to prove Theorem 3.1 we just need to verify the assumptions $\left(\mathrm{A} 1^{\prime}\right),\left(\mathrm{A} 2^{\prime}\right)$ and $\left(\mathrm{A} 3^{\prime}\right)$. Since $b(k)=a([k / n])$, by (A1) we have

$$
b(n-1)=b(n-2)=\cdots=b(0)=a(0)>0
$$

and

$$
\begin{aligned}
b(k) & \in\left[c_{1}\lfloor k / n\rfloor^{-\gamma}, c_{2}\lfloor k / n\rfloor^{-\gamma}\right] \\
& \subset\left[c_{1}(k / n)^{-\gamma}, c_{2}(k /(2 n))^{-\gamma}\right], \quad \forall k \geq n .
\end{aligned}
$$

Let $\gamma^{\prime}:=\gamma, c_{1}^{\prime}:=\min \left\{a(0), c_{1} n^{\gamma}\right\}$, and $c_{2}^{\prime}:=\max \left\{a(0)(n-1)^{\gamma}, c_{2}(2 n)^{\gamma}\right\}$, we can get $\left(\mathrm{A} 1^{\prime}\right)$ holds.

From (A2), (20) and the definition of $\omega_{i}^{j, k}$, we obtain (A2').

By (20), we have

$$
\begin{aligned}
& \left\{(j, i): i \in \mathcal{V}, j \in \mathcal{N}_{i}^{t}\right\} \\
& \quad=\bigcup_{\ln \leq k<(l+1) n}\left\{(j, i): i \in \mathcal{V}, j \in \tilde{\mathcal{N}}_{i}(k)\right\},
\end{aligned}
$$

so $\left(\mathrm{A} 3^{\prime}\right)$ can be deduced from (A3) if we let $k_{s}:=n l_{s}$.

\section{Simulation results and analysis}

\subsection{Clock synchronization algorithm simulation}

Simulation conditions:

1. Number of nodes: $n=10$;

2. Communication cycle: $T=0.01 \mathrm{~s}$;

3. Number of iteration steps: $l=200$;

4. Initial deviation of each node: $\alpha_{i}$ is the random number between $[-T, T]$

5. Communication noise: random number between $[-0.05 T, 0.05 T]$ 
6. Gain function: $a(l)=l /(1+n)$

7. Communication quality: synchronous process under static $(100 \%$ probability of success per communication) and dynamic network (70\%, 40\% and 20\% probability of success per communication).

Simulation results:

Figure 3 shows the convergence curve of the maximum time difference between nodes under the four parameters, and the ordinate is dimensionless value (actual error value/T). It can be seen that with the decline in network quality, the convergence time is slow, but the stable convergence is achieved within 100 steps.

Figure 4 shows the relative error between nodes at the end of simulation (200 steps). It can be seen from the figure that the network communication quality has little influence on the time synchronization error under this algorithm, which reflects the effectiveness of the proposed time synchronization algorithm under the condition of switchable dynamic network.

\subsection{Cooperative guidance simulation}

As shown in Table 1, the speed of five loitering munitions is all set at $30 \mathrm{~m} / \mathrm{s}$ and the maximum normal acceleration at lateral and longitudinal direction is set at $30 \mathrm{~m} / \mathrm{s}^{2}$.

Coordinates of ground fixed targets: $x_{t}=2500, y_{t}=3500, h_{t}=0$.

The parameters of guidance law are: $c_{q}=200^{\circ} / \mathrm{s}, c_{t}=0.5 \mathrm{~s}, N=3, W=30, b=20$, $P=20, Q=5, R=0.1$.

The parameters of clock synchronization algorithm are set as:

1. Number of nodes: $n=5$;

2. Communication cycle: $T=0.005 \mathrm{~s}$;

3. Communication noise: random number between $[-0.05 T, 0.05 T]$;

4. Gain function $a(l)=1 /(1+n)$;

5. Communication quality: dynamic network; the probability of success per communication is $70 \%$.

Fig. 3 Maximum time difference convergence diagram

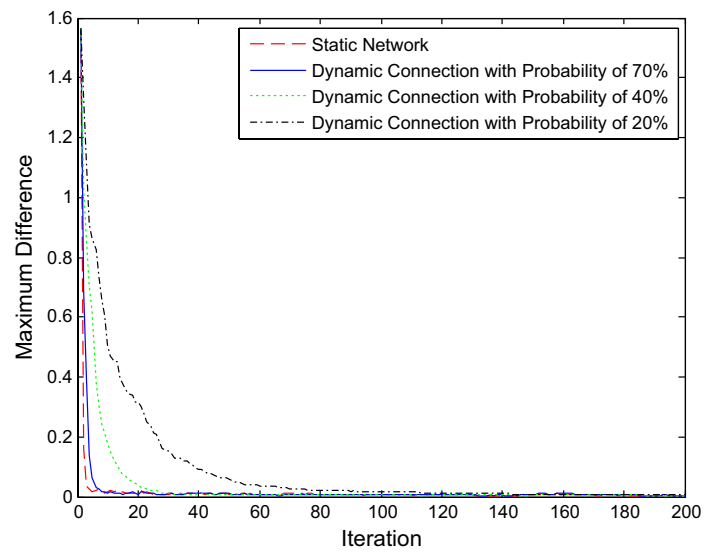




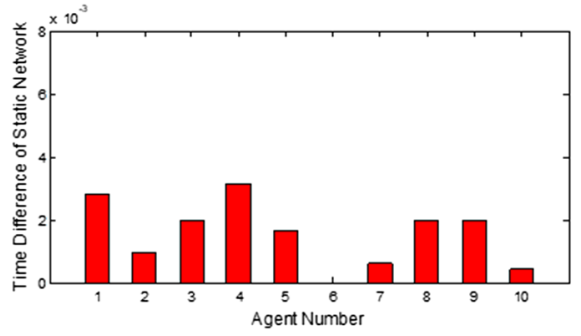

(a) Time Difference of Static Network

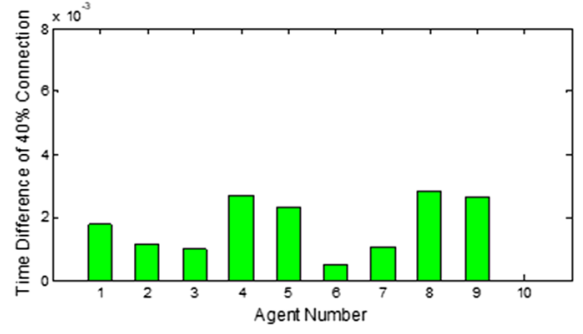

(c) Time Difference of $40 \%$ Connection

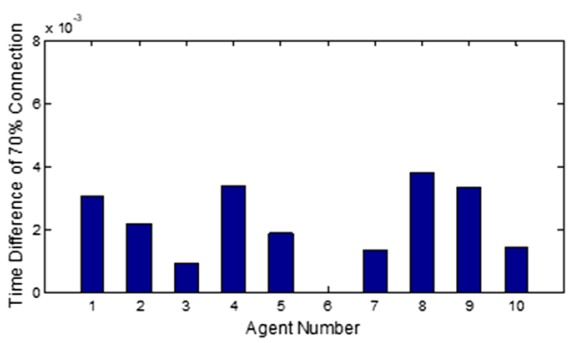

(b) Time Difference of 70\% Connection

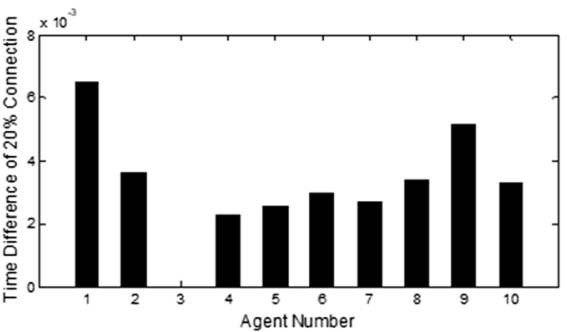

(d) Time Difference of $20 \%$ Connection

Fig. 4 Relative error graph

Table 1 Initial conditions of simulation

\begin{tabular}{lllllc}
\hline $\begin{array}{l}\text { Munition } \\
\text { number }\end{array}$ & Coordinate $x / \mathrm{m}$ & Coordinate $\mathrm{y} / \mathrm{m}$ & Coordinate $\mathrm{h} / \mathrm{m}$ & $\begin{array}{l}\text { Trajectory incli- } \\
\text { nation angle }\left(^{\circ}\right)\end{array}$ & $\begin{array}{l}\text { Trajectory } \\
\text { deflection angle } \\
\left({ }^{\circ}\right)\end{array}$ \\
\hline 1 & 2100 & 3100 & 150 & 0 & -30 \\
2 & 3000 & 3100 & 100 & 0 & -150 \\
3 & 3100 & 3600 & 180 & 0 & 130 \\
4 & 1900 & 3700 & 120 & 0 & 335 \\
5 & 2500 & 2900 & 130 & 0 & 200 \\
\hline
\end{tabular}

\subsubsection{Set terminal attack time as $22 \mathrm{~s}$}

\subsubsection{It does not adopt time synchronization algorithm without initial time} error Table 2 shows the test results. Figure 5 shows the 3D trajectory. Figure 6 shows the overload information of loitering munition. Figure 7 shows the remaining flight time and sliding mode of loitering munition. It can be seen from the simulation results that all the five loitering munitions strike the target with high attack time accuracy, and the attack time error is kept within $0.0005 \mathrm{~s}$, achieving simultaneous arrival. It can be seen from Fig. 6 that the lateral and longitudinal overload of the loitering munition is within the constraint range and is relatively smooth without pathological chattering and other phenomena. In the initial stage of attack, loitering munitions make full use of their overload, and at the end of attack, both lateral and longitudinal 
Table 2 Test result when it does not adopt time synchronization algorithm without initial time error and set the terminal attack time as $22 \mathrm{~s}$

\begin{tabular}{llllll}
\hline Munition number & 1 & 2 & 3 & 4 & 5 \\
\hline Impact time/s (unified timescale) & 21.9996 & 21.9997 & 21.9998 & 21.9995 & 21.9996 \\
Impact time/s (respective timescale) & 21.9996 & 21.9997 & 21.9998 & 21.9995 & 21.9996 \\
\hline
\end{tabular}

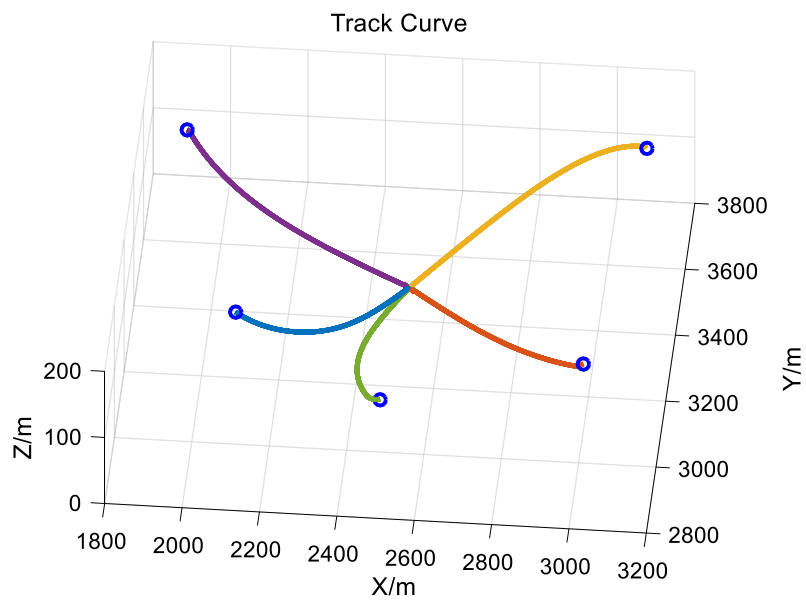

Fig. 5 D trajectory

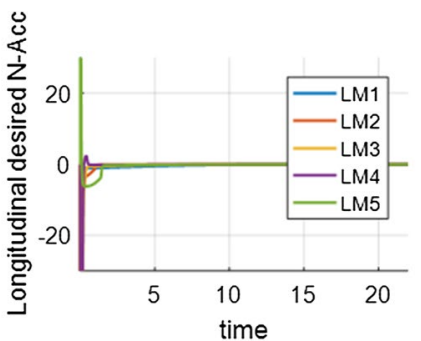

(a) Longitudinal desired N-Acc

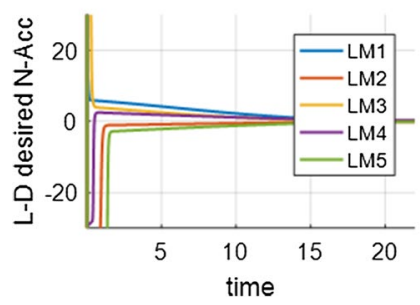

(c) L-D desired N-Acc

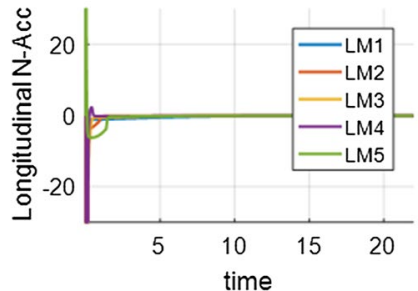

(b) Longitudinal N-Acc

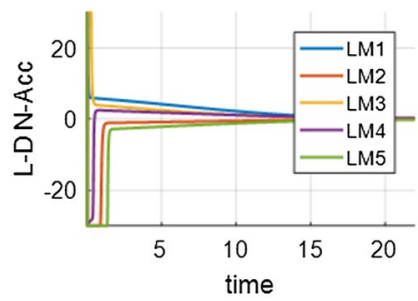

(d) L-D N-Acc

Fig. 6 Overload information of loitering munition (normal acceleration) 


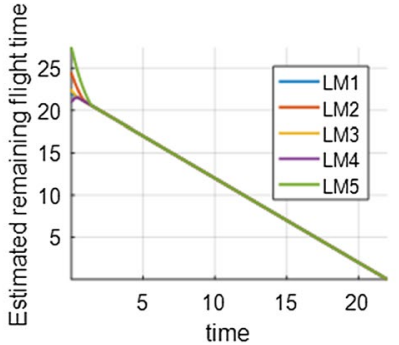

(a) Estimated remaining flight time

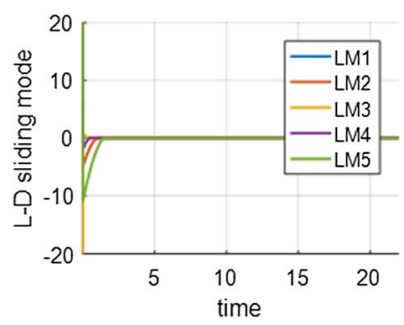

(c) L-D sliding mode

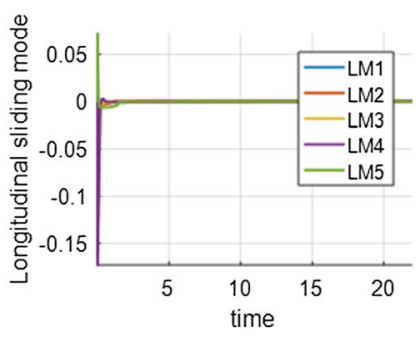

(b) Longitudinal sliding mode

Fig. 7 Remaining flight time and sliding mode of loitering munition

overload approaches 0, with good quality. It can be seen from Fig. 7 that the remaining flight time of each loitering munition quickly converges together and remains until it strikes the target. The lateral and longitudinal sliding mode of the loitering munition has large error at the initial moment and is controlled by the sliding mode controller around steady-state range within several seconds. This indicates that the designed lateral and longitudinal sliding mode guidance law has strong convergence and dynamic characteristics, while the small miss distance and attack time error indicate that the static quality of the controller is better. In addition, since there is no clock error for each loitering munition, the attack time of each loitering munition in the unified timescale is exactly the same in the respective timescale.

5.2.1.2 It does not adopt time synchronization algorithm with initial time error The initial deviation of each node: $\alpha_{i}$ is the random number between [-100T, 100T].

Table 3 shows the test results. Figure 8 shows the 3D trajectory. Figure 9 shows the overload information of loitering munition. Figure 10 shows the remaining flight time and sliding mode of loitering munition. It can be seen from the simulation results that the attack time of the five loitering munitions in their respective timescale is almost exactly the same, close to the set attack time of $22 \mathrm{~s}$. However, due to the different initial time error of loitering munition, the attack time of each loitering munition differs greatly in the unified timescale, about $0.5 \mathrm{~s}$. This situation is shown in Figs. 9 and 10. The initial clock of each loitering munition has error, that is, the start time of simulation curve is different. However, each loitering munition can achieve its precise attack time control, so the time of striking the target is different, 
Table 3 Test result when it doesn't adopt time synchronization algorithm with initial time error and set the terminal attack time as $22 \mathrm{~s}$

\begin{tabular}{llllll}
\hline Munition number & 1 & 2 & 3 & 4 & 5 \\
\hline Impact time/s (unified timescale) & 21.5921 & 21.7732 & 22.0417 & 22.4521 & 22.4595 \\
Impact time/s (respective timescale) & 21.9996 & 21.9997 & 21.9998 & 21.9995 & 21.9996 \\
\hline
\end{tabular}

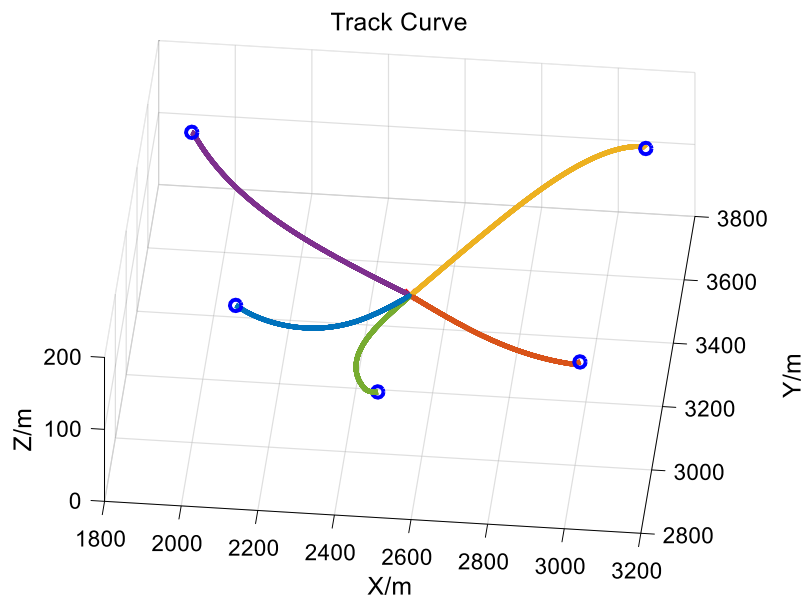

Fig. 8 3D trajectory

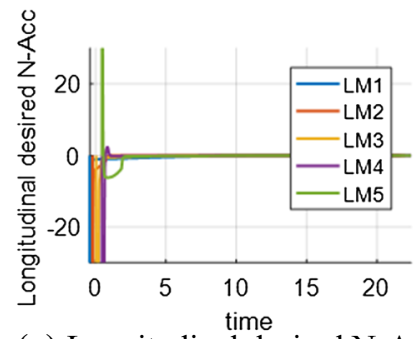

(a) Longitudinal desired N-Acc

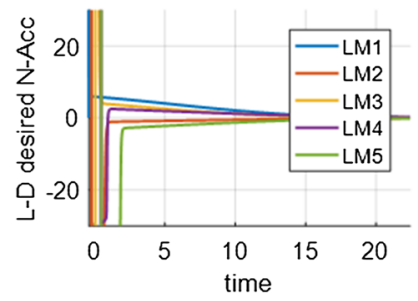

(c) L-D desired N-Acc

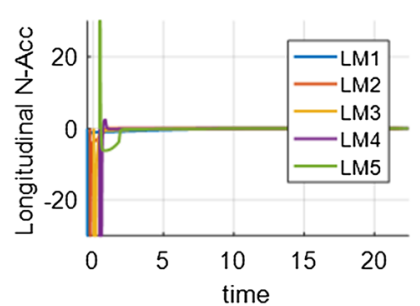

(b) Longitudinal N-Acc

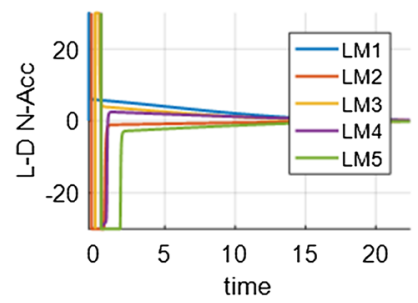

(d) L-D N-Acc

Fig. 9 Overload information of loitering munition 


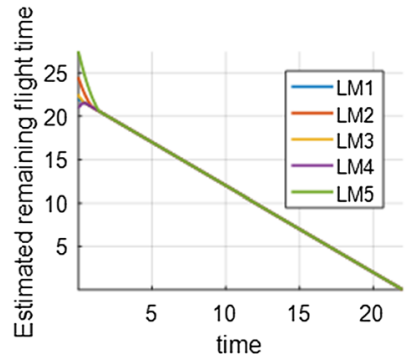

(a) Estimated remaining flight time

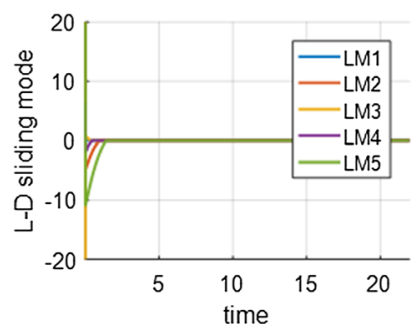

(c) L-D sliding mode

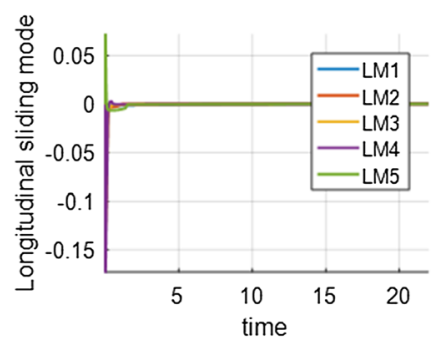

(b) Longitudinal sliding mode

Fig. 10 Remaining flight time and sliding mode of loitering munition

and the time deviation of strike is the initial time deviation between each loitering munition.

5.2.1.3 It adopts time synchronization algorithm with initial time error The initial deviation of each node: $\alpha_{i}$ is the random number between [ $\left.-100 T, 100 T\right]$.

Table 4 shows the test results. Figure 11 shows the 3D trajectory. Figure 12 shows the overload information of loitering munition. Figure 13 shows the remaining flight time and sliding mode of loitering munition. It can be seen from the simulation results that all the five munitions have high strike accuracy and attack time accuracy, and the overload change is gentle. The five loitering munitions have almost exactly the same attack time in different respective timescales, with the difference between them being within $0.0003 \mathrm{~s}$. Although there is an initial time error of the order of $100 \mathrm{~ms}$ between each loitering munition, the timescale of each loitering munition quickly becomes consistent due to the use of time consistency algorithm. In the end, the attack time deviation of each loitering munition is only hundreds of microseconds in the unified timescale, and

Table 4 Test result when it adopts time synchronization algorithm with initial time error and set the terminal attack time as $22 \mathrm{~s}$

\begin{tabular}{llllll}
\hline Munition number & 1 & 2 & 3 & 4 & 5 \\
\hline Impact time/s (unified timescale) & 22.0355 & 22.0356 & 22.0357 & 22.0354 & 22.0355 \\
Impact time/s (respective timescale) & 21.9996 & 21.9997 & 21.9998 & 21.9995 & 21.9996 \\
\hline
\end{tabular}




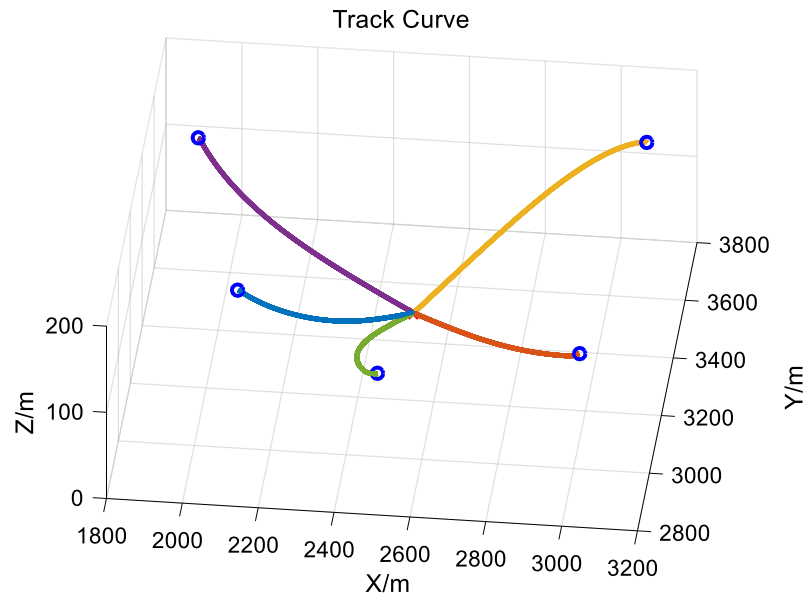

Fig. 11 D trajectory

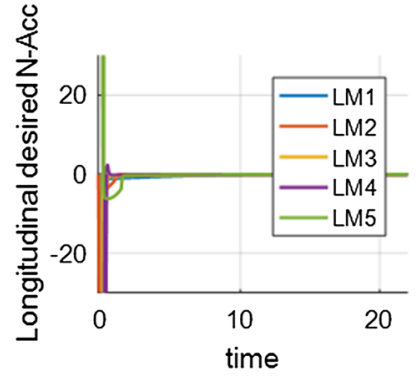

(a) Longitudinal desired N-Acc

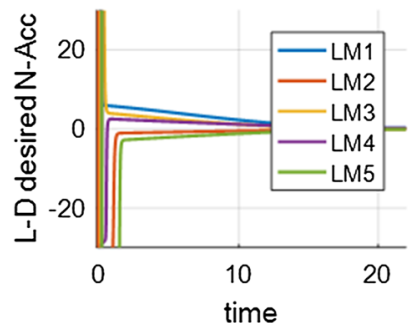

(c) L-D desired N-Acc

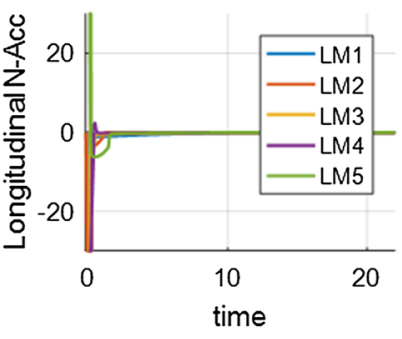

(b) Longitudinal N-Acc

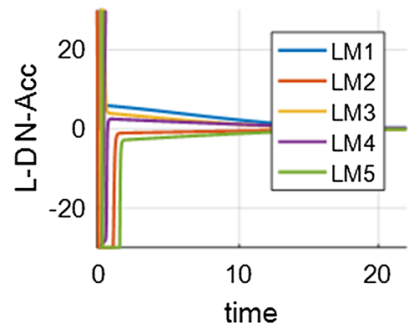

(d) L-D N-Acc

Fig. 12 Overload information of loitering munition

the target can almost be hit simultaneously. Figure 14 shows the maximum clock error of each loitering munition at each time, representing the difference degree of multi-munition clock. It can be seen from the figure that there is a large time difference at the initial time, but it soon converges around 0 , indicating that the 


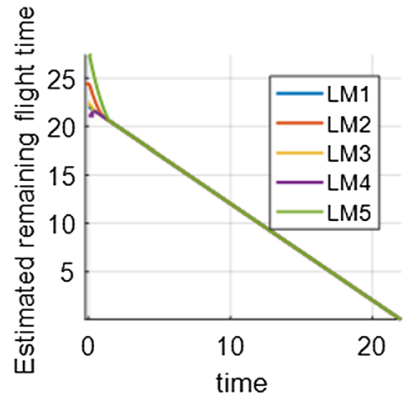

(a) Estimated remaining flight time

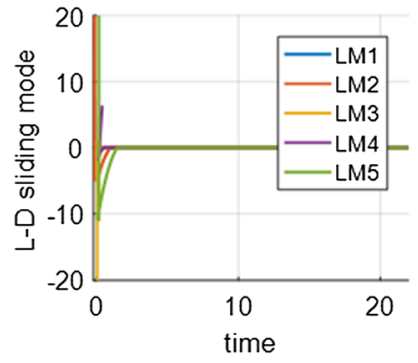

(c) L-D sliding mode

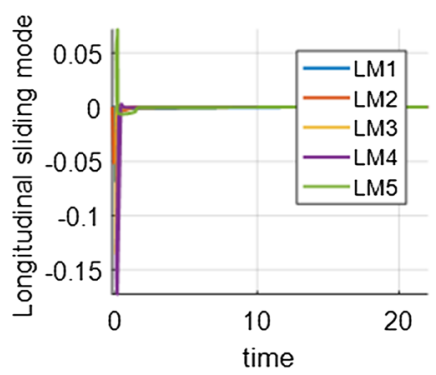

(b) Longitudinal sliding mode

Fig. 13 Remaining flight time and sliding mode of loitering munition

Fig. 14 Maximum time difference curve

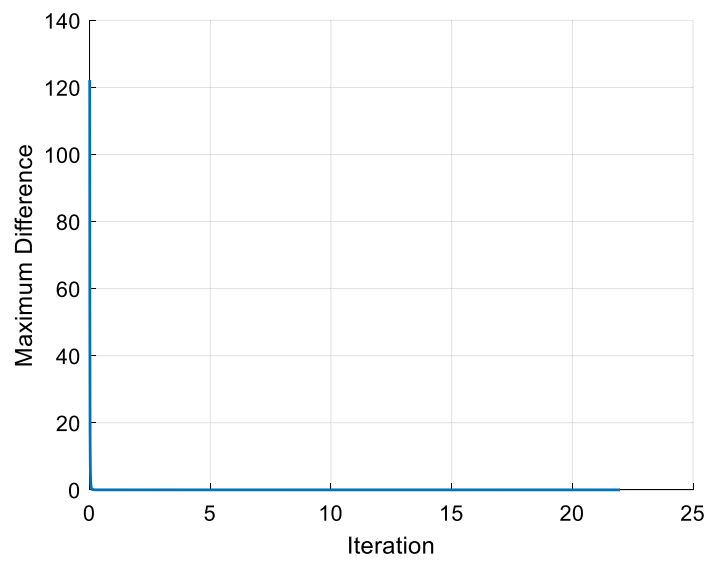

designed clock synchronization algorithm has a better clock registration ability. Figure 15 shows the actual time curve of each loitering munition. Figure 16 shows the dimensionless curve of offset time + offset compensation time of each loitering munition. It can be seen that although each munition has a large offset time error, the offset time error of each munition quickly converges to a certain value through feedback compensation of clock synchronization algorithm and 
Fig. 15 Actual time curve of loitering munition

Fig. 16 (Offset time + offset compensation time) dimensionless curve
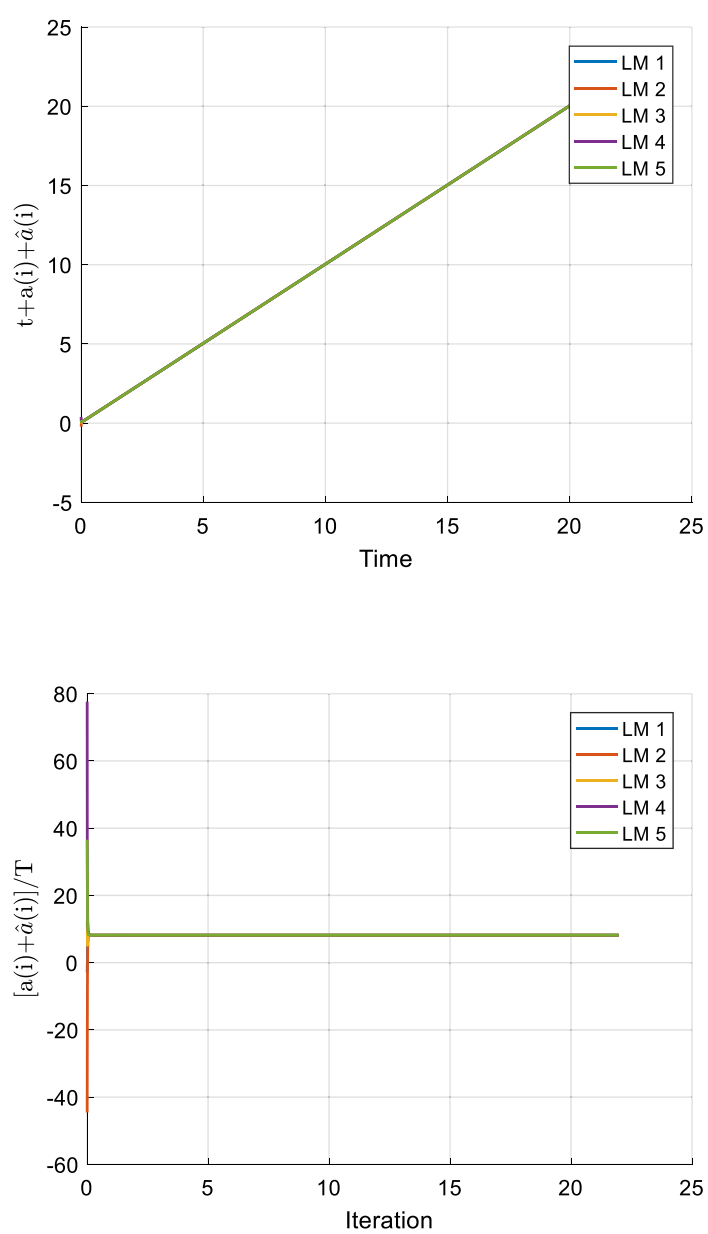

remains close to that value all the time. Figure 17 shows the deviation compensation time curve of each munition. It can be seen that the initial time deviation of each munition is different. By means of clock synchronization algorithm, each munition outputs an appropriate deviation compensation time to eliminate/ reduce inconsistency in the clock. Figure 18 shows the deviation distribution of deviation compensation time of each munition. Select the terminal value of minimum deviation compensation time as the nominal value, differentiate with the deviation compensation time of each other munition and then make them nondimensionalized. And it can be seen that the clock error between each munition can be converged to a small range by this clock synchronization algorithm, which has a good clock synchronization effect. 
Fig. 17 Offset compensation time curve of loitering munition

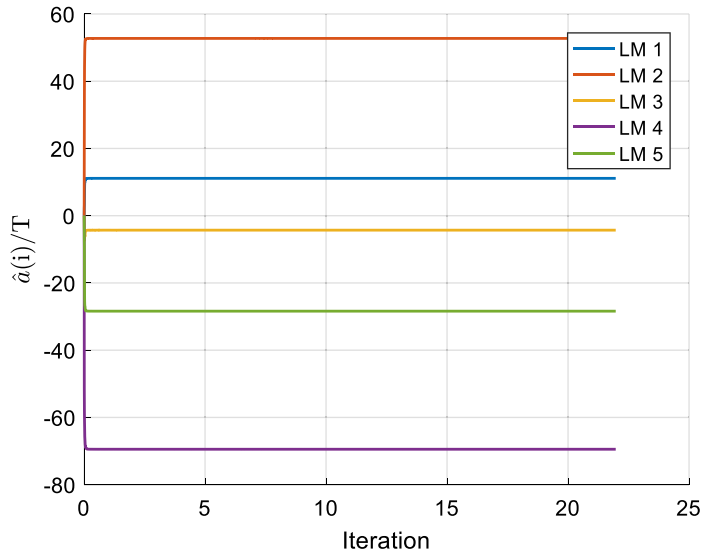

Fig. 18 Loitering munition offset compensation time deviation distribution

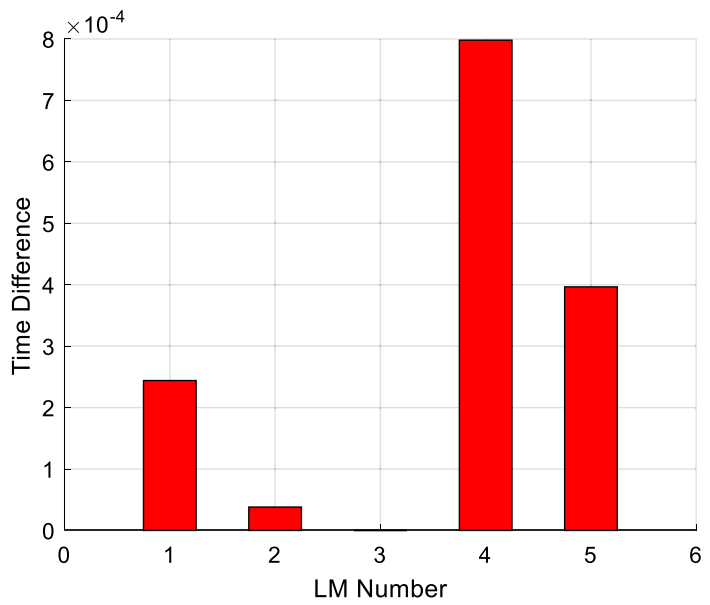

Table 5 Test result when it does not adopt time synchronization algorithm without initial time error and set the terminal attack time as $40 \mathrm{~s}$

\begin{tabular}{llllll}
\hline Munition number & 1 & 2 & 3 & 4 & 5 \\
\hline Impact time/s (unified timescale) & 39.9997 & 39.9994 & 39.9998 & 39.9995 & 39.9996 \\
Impact time/s (respective timescale) & 39.9997 & 39.9994 & 39.9998 & 39.9995 & 39.9996 \\
\hline
\end{tabular}

\subsubsection{Set the terminal attack time as $40 \mathrm{~s}$}

5.2.2.1 It does not adopt time synchronization algorithm without initial time error Table 5 shows the test results. Figure 19 shows the 3D trajectory. It can be seen from the simulation results that all the five loitering munitions strike the target with high attack time accuracy, and the attack time error is kept within hundreds of microseconds, achieving simultaneous arrival. It can be seen from Fig. 20 that the 


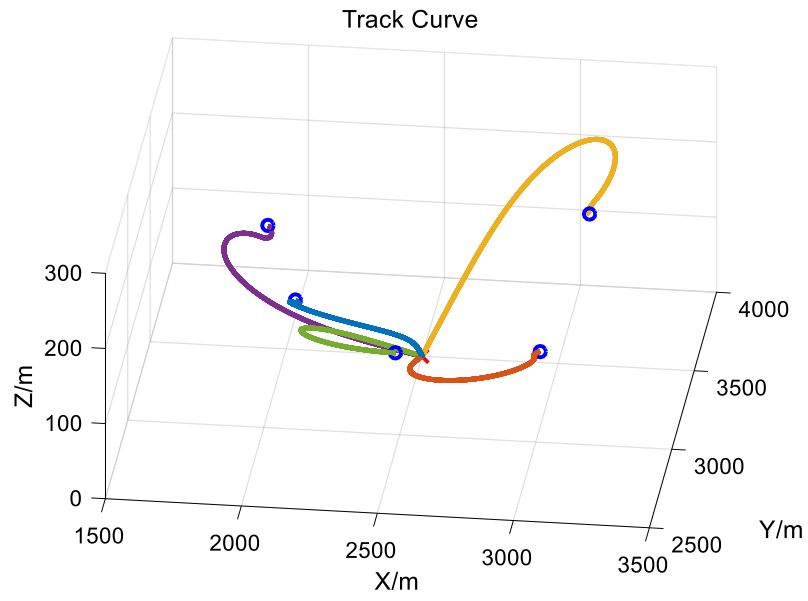

Fig. 19 3D trajectory
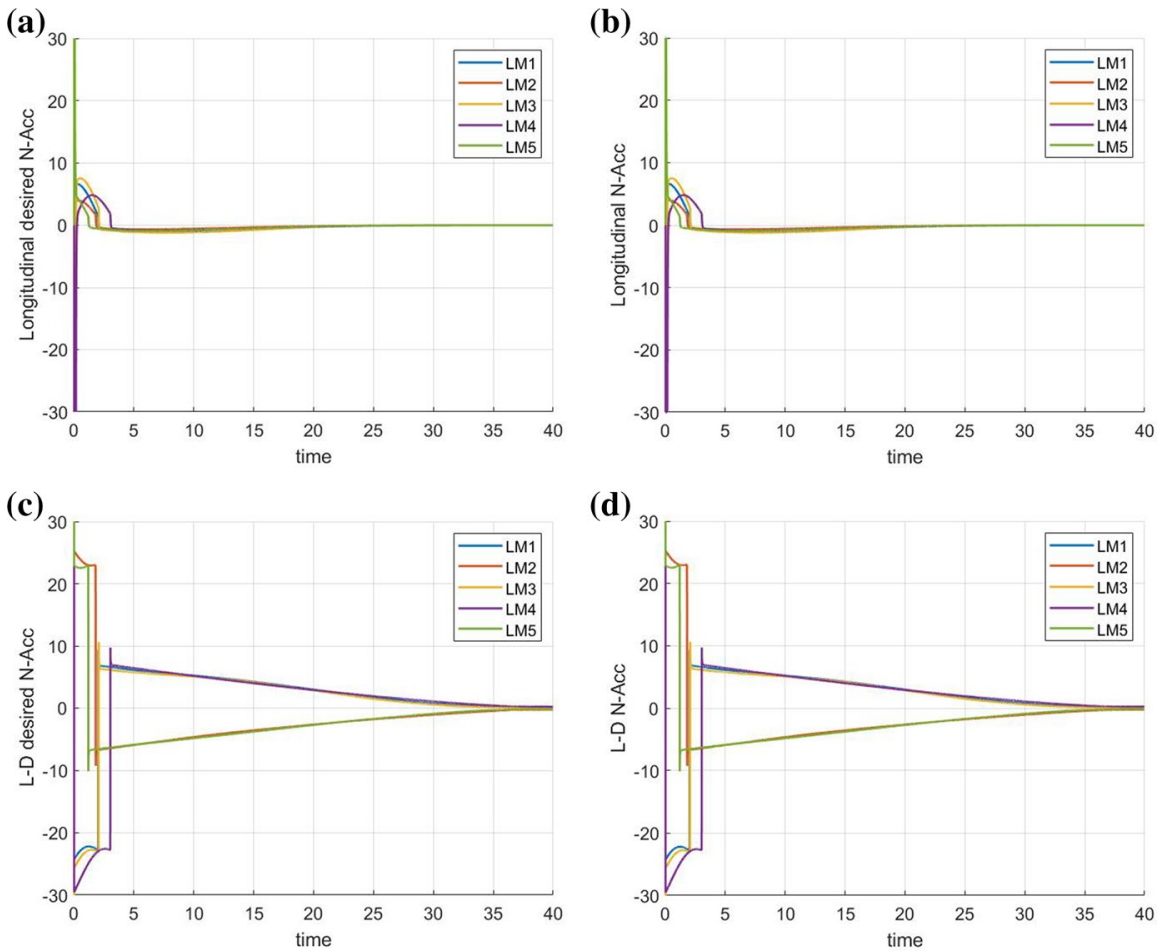

Fig. 20 Overload information of loitering munition

lateral and longitudinal overload of the loitering munition is within the constraint range and is relatively smooth without pathological chattering and other phenomena. 

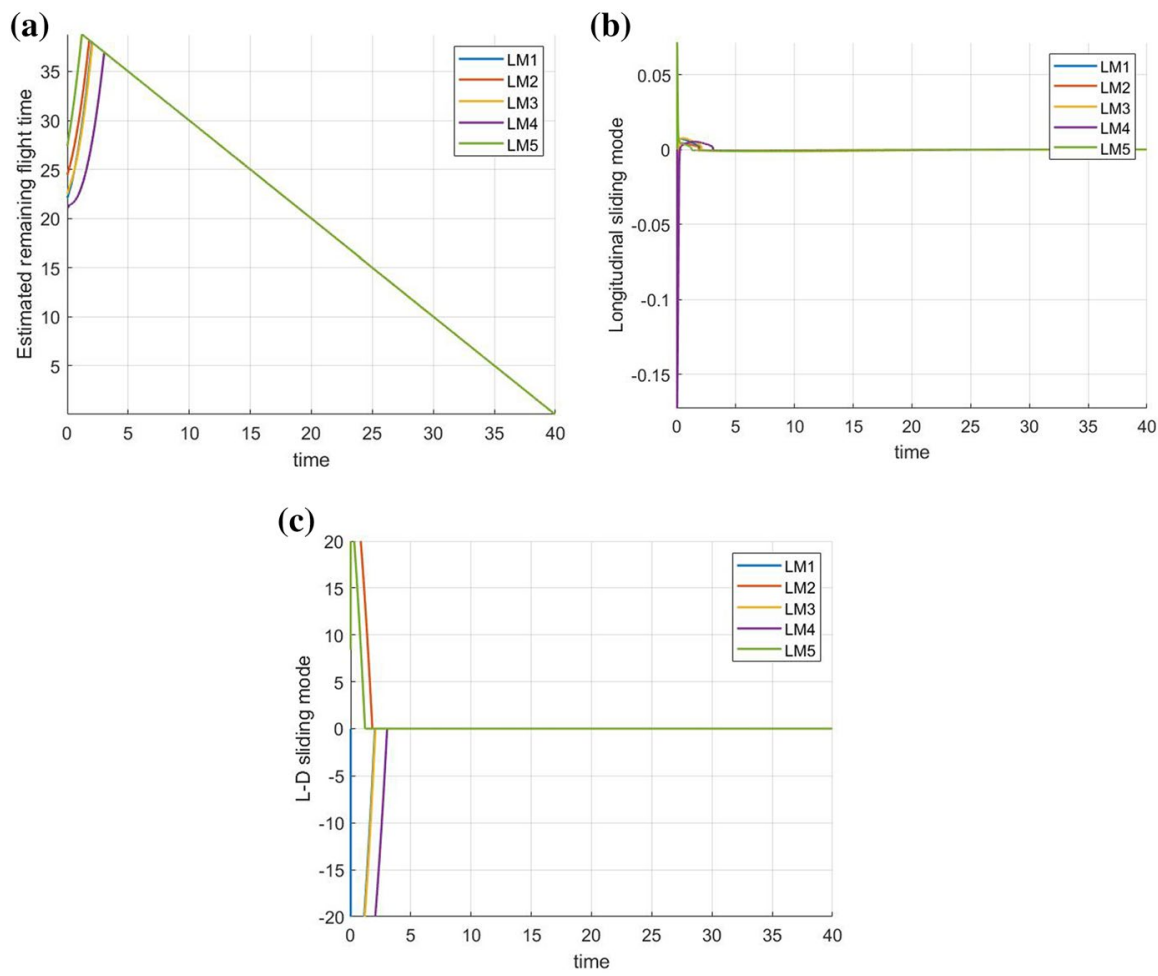

Fig. 21 Remaining flight time and sliding mode of loitering munition

In the initial stage of attack, loitering munitions make full use of their overload, and at the end of attack, both lateral and longitudinal overload approaches 0 , with good quality. It can be seen from Fig. 21 that the remaining flight time of each loitering munition quickly converges together and remains until it strikes the target. The lateral and longitudinal sliding mode of the loitering munition has large error at the initial moment and is controlled by the sliding mode controller around steady-state range within several seconds. This indicates that the designed lateral and longitudinal sliding mode guidance law has strong convergence and dynamic characteristics, while the small miss distance and attack time error indicate that the static quality of the controller is better. In addition, since there is no clock error for each loitering munition, the attack time of each loitering munition in the unified timescale is exactly the same in the respective timescale.

Table 6 Test result when it doesn't adopt time synchronization algorithm with initial time error and set the terminal attack time as $40 \mathrm{~s}$

\begin{tabular}{llllll}
\hline Munition number & 1 & 2 & 3 & 4 & 5 \\
\hline Impact time/s (unified timescale) & 40.0906 & 40.3382 & 39.6428 & 39.7346 & 40.3850 \\
Impact time/s (respective timescale) & 39.9997 & 39.9994 & 39.9998 & 39.9995 & 39.9996 \\
\hline
\end{tabular}




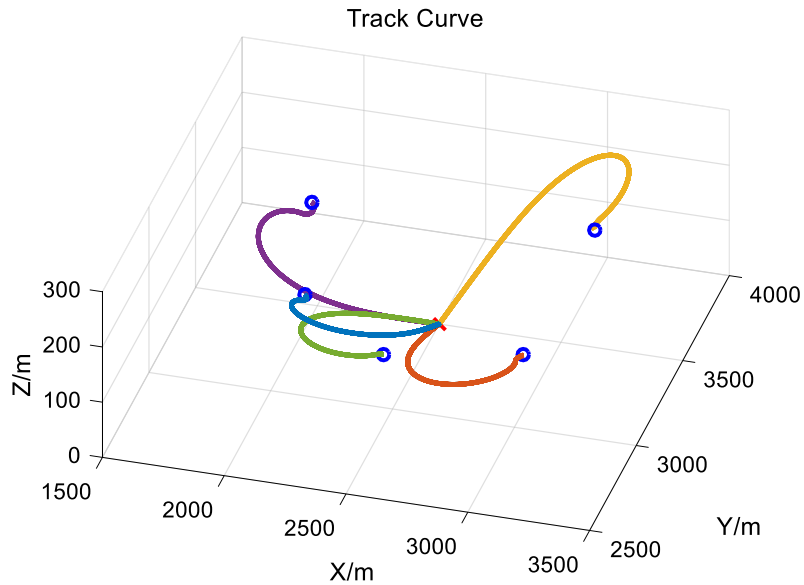

Fig. 22 3D trajectory

(a)

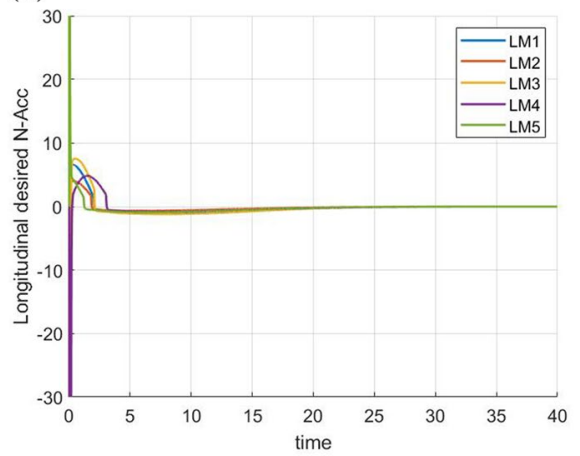

(c)

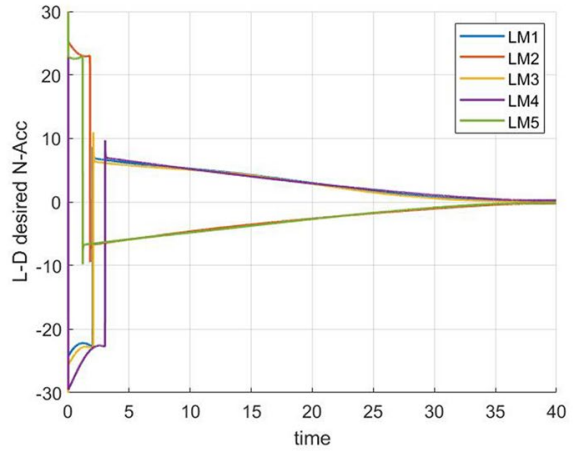

(b)

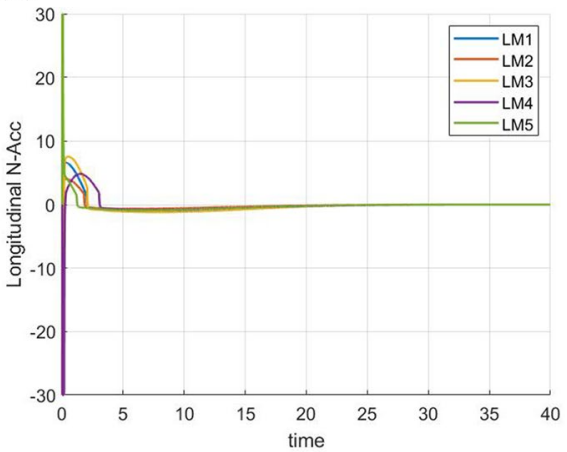

(d)

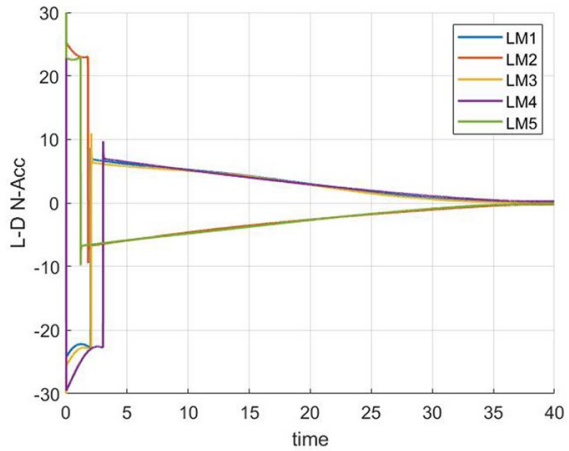

Fig. 23 Overload information of loitering munition 

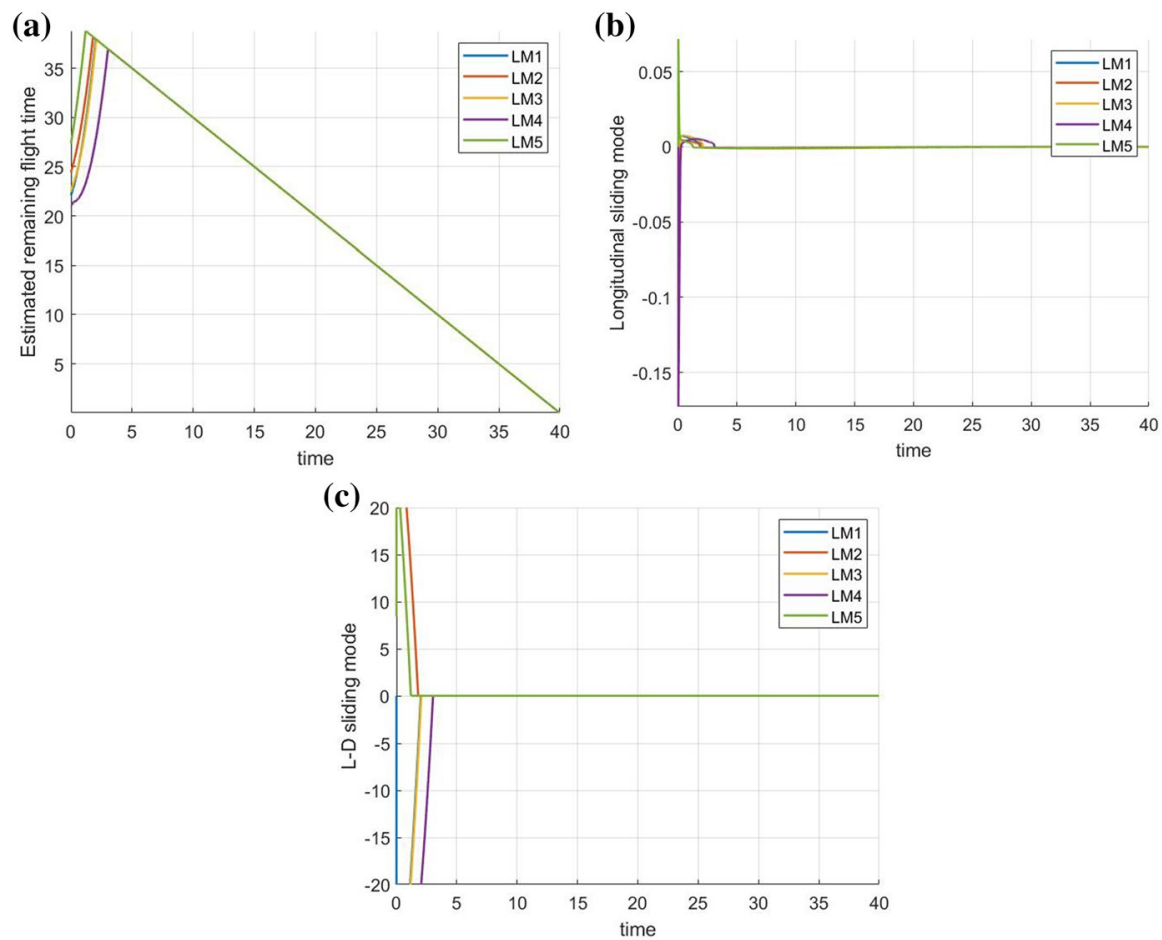

Fig. 24 Remaining flight time and sliding mode of loitering munition

5.2.2.2 It doesn't adopt time synchronization algorithm with initial time error The initial deviation of each node: $\alpha_{i}$ is the random number between $[-100 T, 100 T]$.

Table 6 shows the test results. Figure 22 shows the 3D trajectory. It can be seen from the simulation results that the attack time of the five loitering munitions in their respective timescale is almost exactly the same, close to the set attack time of $40 \mathrm{~s}$. However, due to the different initial time error of loitering munition, the attack time of each loitering munition differs greatly in the unified timescale, about hundreds of milliseconds. This situation is shown in Figs. 23 and 24. Figure 23 shows the overload information of loitering munition. Figure 24 shows the remaining flight time and sliding mode of loitering munition. The initial clock of each loitering munition has error, that is, the start time of simulation curve is different. However, each

Table 7 Test result when it adopts time synchronization algorithm with initial time error and set the terminal attack time as $40 \mathrm{~s}$

\begin{tabular}{llllll}
\hline Munition number & 1 & 2 & 3 & 4 & 5 \\
\hline Impact time/s (unified timescale) & 40.0015 & 40.0012 & 40.0016 & 40.0013 & 40.0014 \\
Impact time/s (respective timescale) & 39.9997 & 39.9994 & 39.9998 & 39.9995 & 39.9996 \\
\hline
\end{tabular}




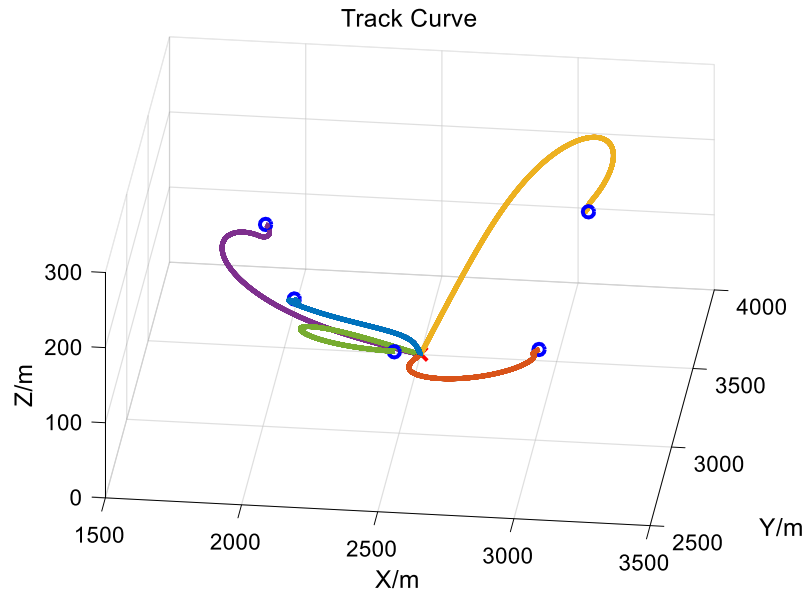

Fig. $253 \mathrm{D}$ trajectory

(a)

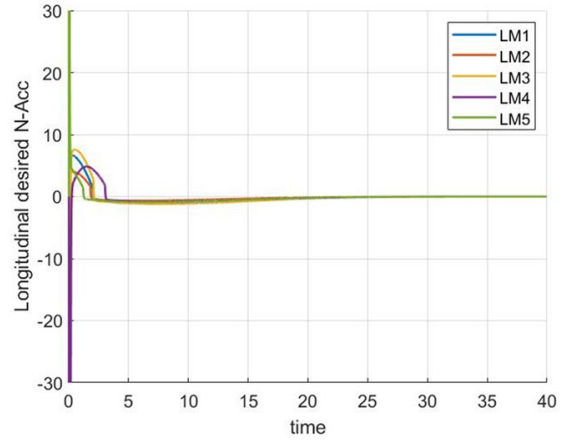

(c)

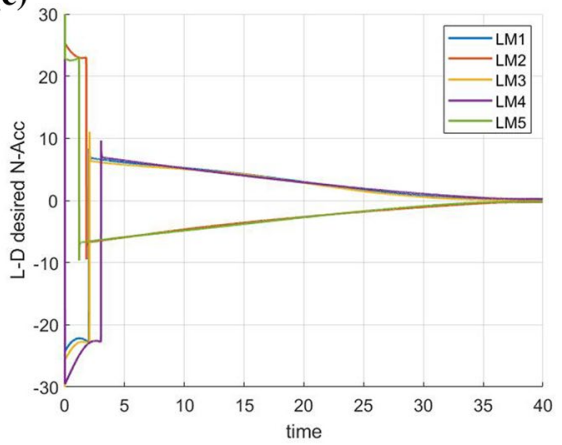

(b)

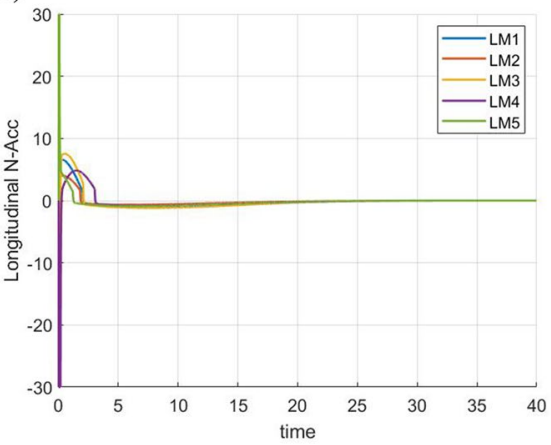

(d)

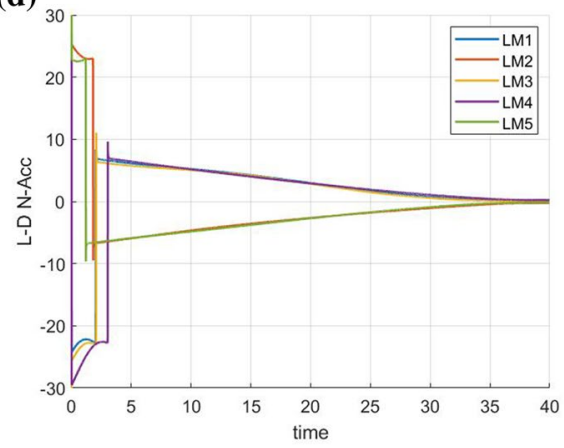

Fig. 26 Overload information of loitering munition 

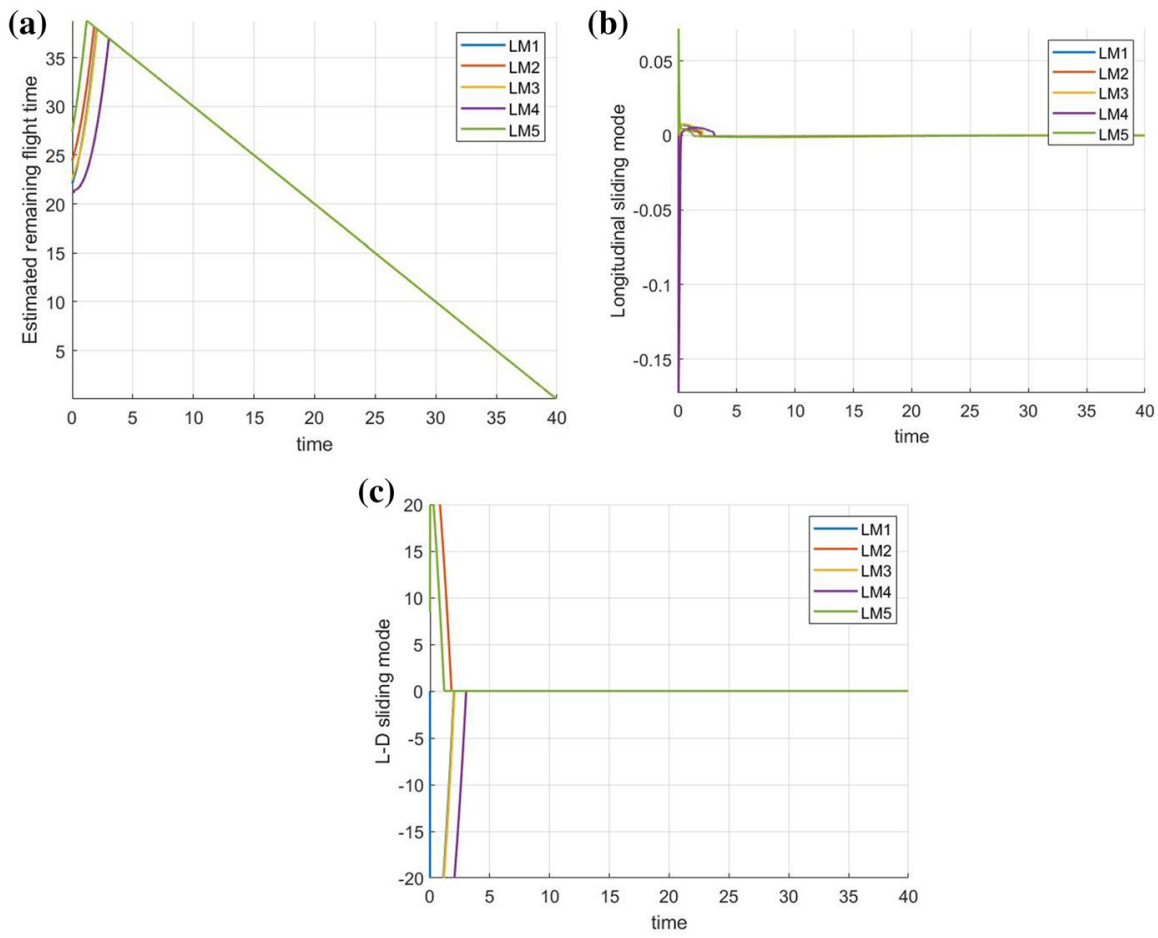

Fig. 27 Remaining flight time and sliding mode of loitering munition

Fig. 28 Maximum time difference curve

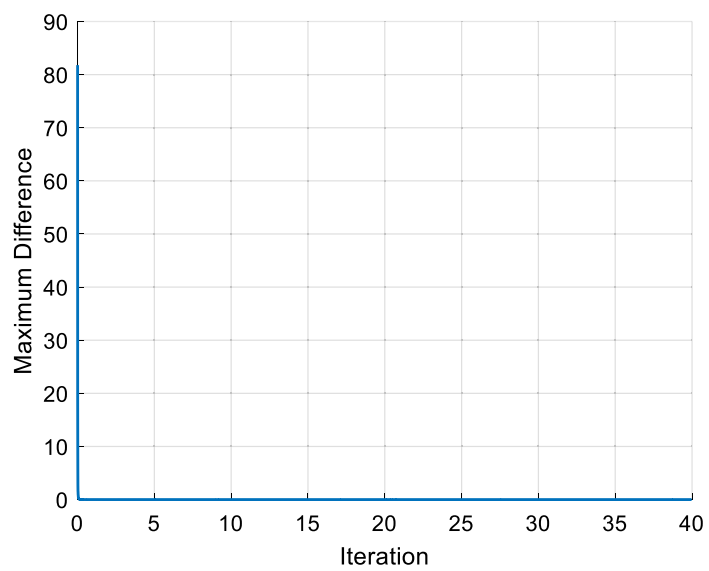

loitering munition can achieve its precise attack time control, so the time of striking the target is different, and the time deviation of strike is the initial time deviation between each loitering munition.

5.2.2.3 It adopts time synchronization algorithm with initial time error Table 7 shows the test results. Figure 25 shows the 3D trajectory. Figure 26 shows the over- 
Fig. 29 Actual time curve of loitering munition

Fig. 30 (Offset time + offset compensation time) dimensionless curve

Fig. 31 Offset compensation time curve of loitering munition
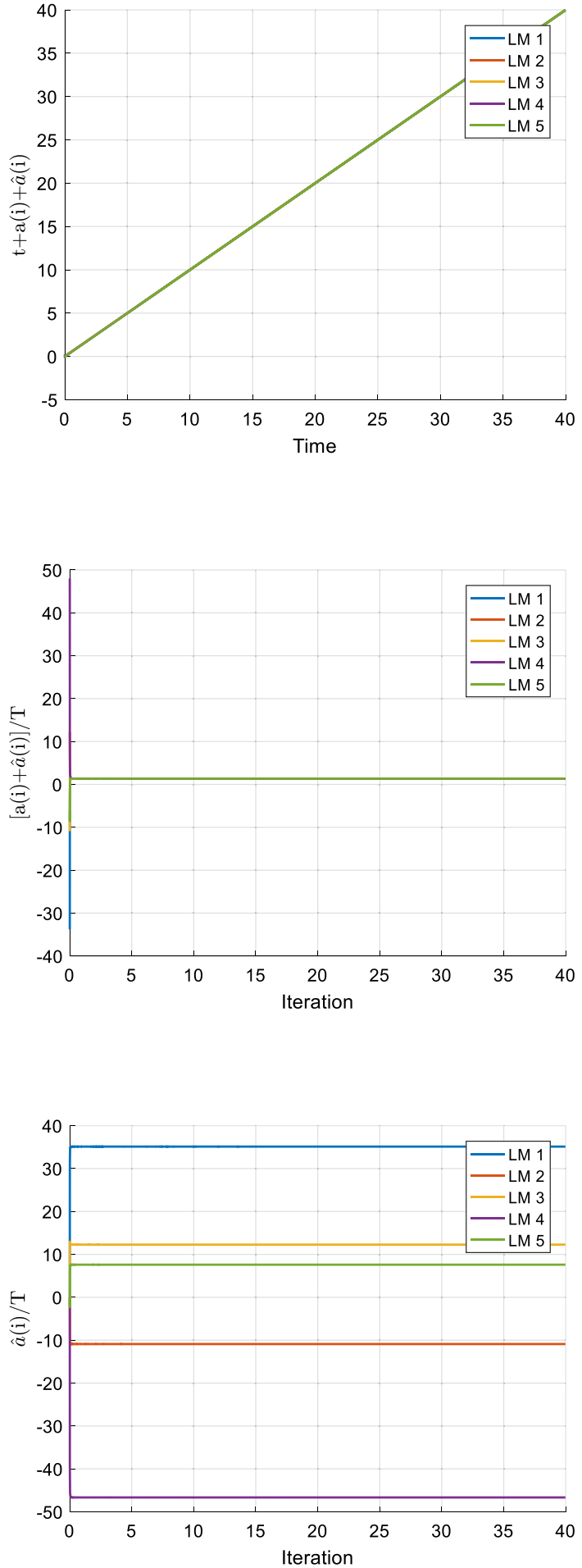
Fig. 32 Loitering munition offset compensation time deviation distribution

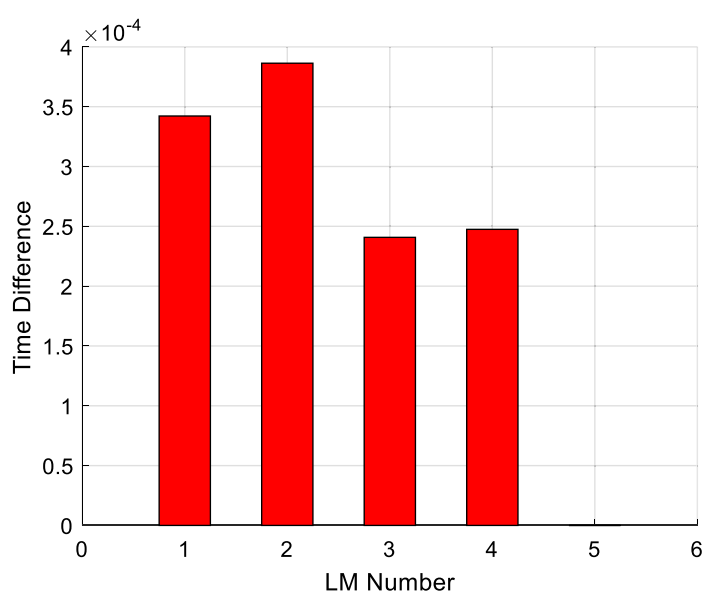

load information of loitering munition. Figure 27 shows the remaining flight time and sliding mode of loitering munition. Figure 28 shows the maximum time difference curve. Figure 29 shows the actual time curve of loitering munition. Figure 30 shows the offset time + offset compensation time) dimensionless curve. Figure 31 shows the offset compensation time curve of loitering munition. Figure 32 shows the loitering munition offset compensation time deviation distribution. It can be seen from the simulation results that all the five munitions have high strike accuracy and attack time accuracy, and the overload change is gentle. The five loitering munitions have almost exactly the same attack time in different respective timescales, around $39.999 \mathrm{~s}$. Although there is an initial time error of tenths of seconds between each loitering munition, the timescale of each loitering munition quickly becomes consistent due to the use of time consistency algorithm. In the end, the attack time deviation of each loitering munition is only hundreds of microseconds in the unified timescale, and the target can almost be hit simultaneously. The results of the time consistency algorithm are similar to those in Sect. 5.2.1.3 and will not be repeated here.

\section{Conclusion}

Based on the above simulation results, it can be seen that the time synchronization algorithm proposed in this paper can synchronize the time of each munition effectively and reliably under different communication masses. The designed longitudinal and lateral guidance law can achieve good coordination and can effectively control the attack time of multiple munitions with different initial parameters while striking the target accurately. The simulation results show that the designed longitudinal and lateral sliding mode guidance law can be combined with the clock synchronization algorithm to compensate for the clock error when there is a certain clock error between each munition, and finally realize the coordinated attack on the target. Given a large range of input parameters and the expected attack time, both high attack accuracy and attack time accuracy can be guaranteed, while the control 
quantity is smooth and approaches 0 at the time of termination, and the sliding mode has no chattering. It shows that the designed controllable guidance law of attack time has good robustness and static dynamic characteristics. In addition, with a large range of expected attack time, the munition can still strike the target with the desired terminal conditions. It shows that the lateral and longitudinal sliding mode guidance law has strong robustness and can adapt to large range of input parameter variation.

Data availability The data used to support the findings of this study are available from the corresponding author upon request.

\section{Compliance with ethical standards}

Conflict of interest The research received no external funding. Among the authors, Sheng Yi and Xiaoyu She are graduate students of Beijing Institute of Technology. The research was performed as part of their education. Jie Li and Chengwei Yang are employed by Beijing Institute of Technology. Dong Guo is employed by Beihang University. Tong Zhao is employed by University of Chinese Academy of Sciences. They only played a supervising role in the research.

Open Access This article is licensed under a Creative Commons Attribution 4.0 International License, which permits use, sharing, adaptation, distribution and reproduction in any medium or format, as long as you give appropriate credit to the original author(s) and the source, provide a link to the Creative Commons licence, and indicate if changes were made. The images or other third party material in this article are included in the article's Creative Commons licence, unless indicated otherwise in a credit line to the material. If material is not included in the article's Creative Commons licence and your intended use is not permitted by statutory regulation or exceeds the permitted use, you will need to obtain permission directly from the copyright holder. To view a copy of this licence, visit http://creativecommons.org/licen ses/by/4.0/.

\section{References}

1. Song J, Song S, Xu S (2017) Three-dimensional cooperative guidance law for multiple missiles with finite-time convergence. Aerosp Sci Technol 67:193-205

2. Wang X, Zhang Y, Liu D, He M (2018) Three-dimensional cooperative guidance and control law for multiple reentry missiles with time-varying velocities. Aerosp Sci Technol 80:127-143

3. Jeon IS, Lee JI, Tahk MJ (2006) Impact-time-control guidance law for anti-ship, missiles. IEEE Trans Control Syst Technol 14(2):260-266

4. Su WS, Li KB, Chen L (2017) Coverage-based cooperative guidance strategy against highly maneuvering target. Aerosp Sci Technol 71:147-155

5. Jeon I-S, Lee J-I, Tahk M-J (2010) Homing guidance law for cooperative attack of multiple missiles. J Guid Control Dyn 33(1):275-280

6. Kumar SR, Ghose D (2015) Impact time guidance for large heading errors using sliding mode control. IEEE Trans Aerosp Electron Syst 51(4):3123-3138

7. Cheng Z, Wang B, Liu L, Wang Y (2018) A composite impact-time-control guidance law and simultaneous arrival. Aerosp Sci Technol 80:403-412

8. Jiang H, An Z, Yu Y, Chen S, Xiong F (2018) Cooperative guidance with multiple constraints using convex optimization. Aerosp Sci Technol 79:426-440

9. Peng C, Liu X, Wu ST et al (2010) Consensus problems in distributed cooperative terminal guidance time of multimissiles. Control Decis 25(10):1557-1561

10. Zhao J, Zhou R (2015) Unified approach to cooperative guidance laws against stationary and maneuvering targets. Nonlinear Dyn 81(4):1635-1647 
11. Wang X, Zhang Y, Wu H (2015) Distributed cooperative guidance of multiple anti-ship missiles with arbitrary impact angle constraint. Aerosp Sci Technol 46:299-311

12. Zhang YA, Ma GX, Wu HL (2014) A biased proportional navigation guidance law with large impact angle constraint and the time-to-go estimation. Proc Inst Mech Eng G J Aerosp Eng 228(10):1725-1734

13. Song L, Zhang Y, Huang D et al (2015) Cooperative simultaneous attack of multi-missiles under unreliable and noisy communication network: a consensus scheme of impact time. Aerosp Sci Technol 47:31-41

14. Hou D, Wang Q, Sun X et al (2015) Finite-time cooperative guidance laws for multiple missiles with acceleration saturation constraints. IET Control Theory Appl 9(10):1525-1535

15. Wei X, Wang Y, Dong S et al (2015) A three-dimensional cooperative guidance law of multimissile system. Int J Aerosp Eng 2015(4):1-8

16. Daughtery E, Qu Z (2014) Optimal design of cooperative guidance law for simultaneous strike. In: Proceedings of the 53rd IEEE Conference on Decision and Control. IEEE Press, Piscataway, pp 988-993

17. Wang Q, Hou D, Li J et al (2014) Consensus analysis of multi-missile decentralized cooperative guidance time with time-delays and topology uncertainty. Acta Armamentarii 35(7):982-989

18. Sun XJ, Zhou R, Hou DL et al (2014) Consensus of leader-followers system of multi-missile with time-delays and switching topologies. Optik 125(3):1202-1208

19. Zhao Q, Dong X, Liang Z, Ren Z (2017) Distributed group cooperative guidance for multiple missiles with fixed and switching directed communication topologies. Nonlinear Dyn 90(4):2507-2523

20. Zhao Q, Dong X, Liang Z, Bai C, Chen J, Ren Z (2017) Distributed cooperative guidance for multiple missiles with fixed and switching communication topologies. Chin J Aeronaut 30(4):1570-1581

21. Huang M (2012) Stochastic approximation for consensus: a new approach via ergodic backward products. IEEE Trans Autom Control 57(12):2994-3008

Publisher's Note Springer Nature remains neutral with regard to jurisdictional claims in published maps and institutional affiliations. 